\title{
On A Class of Time-Varying Gaussian ISI Channels
}

This paper was downloaded from TechRxiv (https://www.techrxiv.org).

\section{LICENSE}

CC BY-NC-SA 4.0

SUBMISSION DATE / POSTED DATE

$13-01-2021 / 15-01-2021$

CITATION

Moshksar, Kamyar (2021): On A Class of Time-Varying Gaussian ISI Channels. TechRxiv. Preprint. https://doi.org/10.36227/techrxiv.13571078.v1

$\mathrm{DOI}$

10.36227/techrxiv.13571078.v1 


\title{
On A Class of Time-Varying Gaussian ISI Channels
}

\author{
Kamyar Moshksar
}

Vancouver, BC, Canada

\begin{abstract}
This paper studies a class of stochastic and time-varying Gaussian intersymbol interference (ISI) channels. The $i^{t h}$ channel tap during time slot $t$ is uniformly distributed over an interval of centre $c_{i}$ and radius $r_{i}$. The array of channel taps is independent along both $t$ and $i$. The channel state information is unavailable at both the transmitter and the receiver. Lower and upper bounds are derived on the White-Gaussian-Input (WGI) capacity $C_{W G I}$ for arbitrary values of the radii $r_{i}$. It is shown that $C_{W G I}$ does not scale with the average input power. The proposed lower bound is achieved by a joint-typicality decoder that is tuned to a set of candidates for the channel matrix. This set forms a net that covers the range of the random channel matrix and its resolution is optimized in order to yield the largest achievable rate. Tools in matrix analysis such as Weyl's inequality on perturbation of eigenvalues of symmetric matrices are used in order to analyze the probability of error.
\end{abstract}

\section{Index Terms}

Intersymbol Interference, Time-Varying Channels, White-Gaussian-Input Capacity, Joint-Typicality Decoding.

\section{INTRODUCTION}

\section{A. Summary of prior art}

Transmission beyond the Nyquist rate over a band-limited communication channel results in a phenomenon known as intersymbol interference (ISI). The time-invariant Gaussian ISI channel is modelled by a linear filter with known finite impulse-response and additive white Gaussian noise [1]. The capacity for this channel was first studied in [2], [21] where it was shown that Gaussian signalling is capacityachieving. Addressing more practical structures for signal transmission, the Gaussian ISI channel with independent and identically distributed (i.i.d.) signalling over a fixed finite alphabet was examined in [3] and more recently in [4], [5]. 
The problem of universal decoding for the Gaussian ISI channel was raised for the first time in [6]. Roughly speaking, a universal decoder is one whose structure does not depend on the channel parameters and yet it achieves the same error exponent as the optimum maximum likelihood decoder tuned to the actual channel parameters. Influenced by the pioneering work of Ziv in [7] on universal decoding over channels with memory, the authors in [8] coined various notions of universal decoding such as random vs. deterministic and weak vs. strong universal decoding. In particular, [8] provided an answer to the problem raised in [6]. The authors showed that if the receiver knows the length and an upper bound on the energy of the channel impulse response, the Gaussian ISI channel admits a universal decoder in the strong sense. Later in [9], another universal decoder was proposed from the viewpoint of composite hypothesis testing. More recently, a universal decoder was presented in [10] which is easier to evaluate compared to the previously reported decoders in [8], [9].

The time-varying Gaussian ISI channel is less studied in the literature. Reference [11] presented information-theoretic considerations for multi-carrier transmission in time-varying Gaussian ISI channels. Reference [12] derived the capacity of block-memoryless channels with block-memoryless side information. Subsequently, the capacity of time-varying Gaussian ISI channels was characterized. Both [11] and [12] assume that perfect channel state information about the time-varying channel is available at the receiver side.

\section{B. Summary of contributions}

We study a class of stochastic and time-varying Gaussian ISI channels where the transmitter and the receiver only know the statistics of the array of channel taps. Consider a Gaussian ISI channel initially modelled by a sequence of filter taps $c_{0}, c_{1}, \cdots, c_{k}$ as its impulse response. Let $\boldsymbol{h}_{t, i}$ be the actual $i^{\text {th }}$ channel tap during time slot $t$. Then the initial model assumes $\boldsymbol{h}_{t, i}=c_{i}$ for all $t$ and $i$. We ask the following question:

If $\boldsymbol{h}_{t, i}$ is not exactly the constant $c_{i}$, but a random variable that takes values near $c_{i}$, then how would that impact the channel capacity?

In an attempt to answer this question, it is assumed that $\boldsymbol{h}_{t, i}$ is uniformly distributed on the interval $\left[c_{i}-r_{i}, c_{i}+r_{i}\right]$ for all $t$ and $i$ where $r_{i} \geq 0$ is a given constant. Moreover, the array $\boldsymbol{h}_{t, i}$ is independent along both indices $t$ and $i$. For tractability reasons, the focus of the paper is on the largest rate that can be achieved by employing white Gaussian codebooks. This is referred to as the White Gaussian Input (WGI) capacity denoted by $C_{W G I}$. The main contributions of the paper are lower and upper bounds on $C_{W G I}$. The 
proposed upper bound shows that $C_{W G I}$ does not scale with the average input power. The proposed lower bound is attained by a joint-typicality decoder that is tuned to a net of candidates for the channel matrix during the transmission of a message. This net covers the ambiguity region for the random channel matrix and its resolution is adjusted in order to yield the largest achievable rate. Tools in matrix analysis such as Weyl's inequality on perturbation of eigenvalues of symmetric matrices are used in order to analyze the probability of error.

The rest of the paper is organized as follows. We end the current section by listing the notations and presenting a lemma on the volume of Gaussian typical sets that improves upon the existing estimates in the literature. System model, the problem statement and a summary of main results appear in Section II. Section III offers examples to compare the lower and upper bounds on $C_{W G I}$. Section IV describes the structure of the proposed decoder. Section V is devoted to error analysis where we outline the proof of the main result (Theorem 1). The majority of details are referred to the appendices at the end of the paper. Finally, Section VI concludes the paper.

\section{Notations}

For a real number $x, x^{+}=\max \{0, x\}$ and $[x]$ is the closest integer to $x$. The Euclidean space of sequences of length $n$ whose entries are real numbers is denoted by $\mathbb{R}^{n}$. Vectors and sequences are identified by an underline such as $\underline{x}$. Random quantities appear in bold such as $\boldsymbol{x}$ and $\underline{\boldsymbol{x}}$ with realizations $x$ and $\underline{x}$, respectively. An $m \times n$ matrix whose all entries are zeros is denoted by $0_{m, n}$. We use $\underline{0}_{n}$ to denote a column vector of length $n$ whose all entries are zeros. The $n \times n$ identity matrix is denoted by $I_{n}$. The transpose, inverse, trace and determinant of a square matrix $A$ are denoted by $A^{T}, A^{-1}, \operatorname{tr}(A)$ and $\operatorname{det}(A)$, respectively. The entry at the $i^{\text {th }}$ row and the $j^{\text {th }}$ column of a matrix $A$ is denoted by $A_{i, j}$ or $[A]_{i, j}$. The $i^{\text {th }}$ entry of a vector $\underline{x}$ is denoted by $x_{i}$ or $[\underline{x}]_{i}$. The 2-norm (Frobenius norm) and the spectral norm (operator norm) of a matrix $A$ are defined by

$$
\|A\|_{2}:=\left(\operatorname{tr}\left(A^{T} A\right)\right)^{1 / 2}
$$

and

$$
\|A\|:=\max _{\|\underline{x}\|_{2} \leq 1}\|A \underline{x}\|_{2}=\left(\lambda_{\max }\left(A^{T} A\right)\right)^{\frac{1}{2}}
$$


respectively, where $\lambda_{\max }(M)$ denotes the largest eigenvalue of a symmetric matrix $M$. The probability law of a random variable $\boldsymbol{x}$ (the induced probability measure on the range of $\boldsymbol{x}$ ) is denoted by $\mathcal{L}_{\boldsymbol{x}}(\cdot)$. The probability density function (PDF) of a continuous random variable $\underline{\boldsymbol{x}}$ is denoted by $p_{\boldsymbol{x}}(\cdot)$. The PDF of a Gaussian random vector of length $n$ with zero mean and covariance matrix $\Sigma$ is denoted by

$$
p_{\mathrm{G}}(\underline{x} ; \Sigma):=\frac{1}{(2 \pi)^{\frac{n}{2}} \sqrt{\operatorname{det}(\Sigma)}} \exp \left(-\frac{1}{2} \underline{x}^{T} \Sigma^{-1} \underline{x}\right) .
$$

We write $\underline{\boldsymbol{x}} \sim \mathrm{N}\left(\underline{0}_{n}, \Sigma\right)$ if $p_{\underline{x}}(\underline{x})=p_{\mathrm{G}}(\underline{x} ; \Sigma)$. The differential entropy of a random vector $\underline{\boldsymbol{x}} \sim \mathrm{N}\left(\underline{0}_{n}, \Sigma\right)$ is denoted by $h_{\mathrm{G}}(\Sigma)$, i.e.,

$$
h_{\mathrm{G}}(\Sigma):=\frac{1}{2} \log \left((2 \pi e)^{n} \operatorname{det}(\Sigma)\right)
$$

where $\log (\cdot)$ is the logarithm function with base 2 . The underlying probability measure is denoted by $\operatorname{Pr}(\cdot)$. Finally, $\mathbb{E}[\cdot]$ denotes the expectation operator against $\operatorname{Pr}(\cdot)$.

\section{A lemma on the volume of Gaussian typical sets}

Let us recall the definition for a typical set [13], [20]. For $\eta>0$, positive integer $n$ and an $n \times n$ positive-definite matrix $\Sigma$, the Gaussian typical set $\mathcal{T}_{\eta}^{(n)}(\Sigma)$ is defined by

$$
\begin{aligned}
\mathcal{T}_{\eta}^{(n)}(\Sigma) & :=\left\{\underline{a} \in \mathbb{R}^{n}:\left|\frac{1}{n} \log p_{\mathrm{G}}(\underline{a} ; \Sigma)+\frac{1}{n} h_{\mathrm{G}}(\Sigma)\right|<\frac{\eta}{2 \ln 2}\right\} \\
& =\left\{\underline{a} \in \mathbb{R}^{n}:\left|\frac{1}{n} \underline{a}^{T} \Sigma^{-1} \underline{a}-1\right|<\eta\right\} .
\end{aligned}
$$

The following lemma on the volume of a typical set will be used later in the paper:

Lemma 1. The volume of the typical set $\mathcal{T}_{\eta}^{(n)}(\Sigma)$ is bounded from above by

$$
\operatorname{Vol}\left(\mathcal{T}_{\eta}^{(n)}(\Sigma)\right) \leq 2^{h_{\mathrm{G}}(\Sigma)+\frac{n}{2} \log (1+\eta)}
$$

Proof. See Appendix A. Reference [13] reports the upper bound $2^{h_{\mathrm{G}}(\Sigma)+\frac{n}{2 \ln 2} \eta}$ on $\operatorname{Vol}\left(\mathcal{T}_{\eta}^{(n)}(\Sigma)\right)$. The bound in (6) is tighter than $2^{h_{\mathrm{G}}(\Sigma)+\frac{n}{2 \ln 2} \eta}$. The proposed lower bound on $C_{W G I}$ in Theorem 1 benefits from this improvement. 


\section{SYSTEM MODEL AND SUMMARY OF MAIN RESULTS}

The stochastic and time-varying Gaussian ISI channel with memory-depth $k \geq 1$ is defined by

$$
\boldsymbol{y}_{t}=\sum_{i=0}^{k} \boldsymbol{h}_{t, i} \boldsymbol{x}_{t-i}+\boldsymbol{z}_{t}, \quad t=1,2, \cdots
$$

where $\boldsymbol{h}_{t, 0}, \boldsymbol{h}_{t, 1}, \cdots, \boldsymbol{h}_{t, k}$ are the channel coefficients (taps) during time slot $t=1,2, \cdots$ and $\boldsymbol{x}_{t}, \boldsymbol{y}_{t}$ and $\boldsymbol{z}_{t}$ are the channel input, the channel output and the additive noise at time slot $t$, respectively. The process $\boldsymbol{z}_{1}, \boldsymbol{z}_{2}, \cdots$ is white Gaussian with zero mean and unit variance. The input process $\boldsymbol{x}_{1}, \boldsymbol{x}_{2}, \cdots$ is subject to the average power constraint

$$
\frac{1}{n} \sum_{t=1}^{n}\left|\boldsymbol{x}_{t}\right|^{2} \leq P
$$

where $n$ is the length of a codeword.

The following assumptions are made on the array of channel coefficients:

(i) The array of channel taps $\boldsymbol{h}_{t, i}$ is independent along both indices $t$ and $i$ with uniform marginals. More precisely, there is a sequence of numbers $\underline{c}=\left(c_{0}, c_{1}, \cdots, c_{k}\right)$ and a sequence of nonnegative numbers $\underline{r}=\left(r_{0}, r_{1}, \cdots, r_{k}\right)$ such that $\boldsymbol{h}_{t, i}$ is uniformly distributed over the interval $\left[c_{i}-r_{i}, c_{i}+r_{i}\right]$ for every $t, i$. We define

$$
c_{s}:=\sum_{i=0}^{k}\left|c_{i}\right|, \quad r_{s}:=\sum_{i=0}^{k} r_{i}
$$

(ii) The transmitter and the receiver only know the distribution of the random variables $\boldsymbol{h}_{t, i}$.

(iii) The processes $\boldsymbol{h}_{t, i}$ and $\boldsymbol{z}_{t}$ are independent.

To describe the ISI channel in matrix form, assume a codeword is transmitted during time slots $t=$ $1, \cdots, n$. Define

$$
m:=n+k
$$

and

$$
\underline{\boldsymbol{x}}:=\left[\begin{array}{llll}
\boldsymbol{x}_{1} & \boldsymbol{x}_{2} & \cdots & \boldsymbol{x}_{n}
\end{array}\right]^{T}, \quad \underline{\boldsymbol{y}}:=\left[\begin{array}{llll}
\boldsymbol{y}_{1} & \boldsymbol{y}_{2} & \cdots & \boldsymbol{y}_{m}
\end{array}\right]^{T}, \quad \underline{\boldsymbol{z}}:=\left[\begin{array}{llll}
\boldsymbol{z}_{1} & \boldsymbol{z}_{2} & \cdots & \boldsymbol{z}_{m}
\end{array}\right]^{T} .
$$


Then

$$
\underline{\boldsymbol{y}}=\boldsymbol{H} \underline{\boldsymbol{x}}+\underline{\boldsymbol{z}},
$$

where the $m \times n$ channel matrix $\boldsymbol{H}$ is given by

$$
\boldsymbol{H}_{i, j}:=\left\{\begin{array}{cc}
\boldsymbol{h}_{i, i-j} & 0 \leq i-j \leq k \\
0 & \text { otherwise }
\end{array} .\right.
$$

For example, when $n=4$ and $k=2$, we have $m=4+2=6$ and the matrices $\boldsymbol{H}$ and $\mathbb{E}[\boldsymbol{H}]$ are given by

$$
\boldsymbol{H}=\left[\begin{array}{cccc}
\boldsymbol{h}_{1,0} & 0 & 0 & 0 \\
\boldsymbol{h}_{2,1} & \boldsymbol{h}_{2,0} & 0 & 0 \\
\boldsymbol{h}_{3,2} & \boldsymbol{h}_{3,1} & \boldsymbol{h}_{3,0} & 0 \\
0 & \boldsymbol{h}_{4,2} & \boldsymbol{h}_{4,1} & \boldsymbol{h}_{4,0} \\
0 & 0 & \boldsymbol{h}_{5,2} & \boldsymbol{h}_{5,1} \\
0 & 0 & 0 & \boldsymbol{h}_{6,2}
\end{array}\right], \quad \mathbb{E}[\boldsymbol{H}]=\left[\begin{array}{cccc}
c_{0} & 0 & 0 & 0 \\
c_{1} & c_{0} & 0 & 0 \\
c_{2} & c_{1} & c_{0} & 0 \\
0 & c_{2} & c_{1} & c_{0} \\
0 & 0 & c_{2} & c_{1} \\
0 & 0 & 0 & c_{2}
\end{array}\right] .
$$

The message $\boldsymbol{W}$ is uniformly distributed over the message set $\left\{1,2, \cdots, 2^{n R}\right\}$ where $R$ is the transmission rate. The encoder maps $\boldsymbol{W}$ to a codeword $\underline{\boldsymbol{x}}$ inside the codebook $\mathcal{C}$. This codeword is then transmitted over the channel in (12) during time slots $t=1, \cdots, n$. The decoder receives the vector $\underline{\boldsymbol{y}}$ and generates the estimate $\widehat{\boldsymbol{W}}$ for $\boldsymbol{W}$. The probability of decoding error is denoted by

$$
p_{e}(\mathcal{C}):=\operatorname{Pr}(\widehat{\boldsymbol{W}} \neq \boldsymbol{W})
$$

Next, we define the notion of white-Gaussian-input capacity.

Definition- Let $\mathscr{C}$ be the ensemble of codebooks whose $2^{n R}$ codewords are realizations of independent $\mathrm{N}\left(\underline{0}_{n}, P I_{n}\right)$ random vectors. We say a transmission rate $R$ is White-Gaussian-Input achievable (WGIachievable), if

$$
\lim _{n \rightarrow \infty}\left\langle p_{e}(\mathcal{C})\right\rangle_{\mathscr{C}}=0
$$

where $\left\langle p_{e}(\mathcal{C})\right\rangle_{\mathscr{C}}$ denotes the average of $p_{e}(\mathcal{C})$ as the codebook $\mathcal{C}$ runs over the ensemble $\mathscr{C}$. The supremum of all WGI-achievable rates is denoted by $C_{W G I}$ and referred to as the WGI-capacity. $\diamond$

In simple words, $C_{W G I}$ is the largest rate that can be achieved by white Gaussian codewords. In the 
special case where the ISI channel is deterministic and time-invariant, i.e., $r_{i}=0$ for every $i$, it is well-known [2] that the WGI-capacity denoted by $C_{W G I, 0}(P)$ is given by

$$
C_{W G I, 0}(P):=\left.C_{W G I}\right|_{\forall i \geq 0, r_{i}=0}=\frac{1}{4 \pi} \int_{0}^{2 \pi} \log \left(1+\left|\sum_{i=0}^{k} c_{i} e^{\sqrt{-1} i \omega}\right|^{2} P\right) d \omega
$$

The main contributions of this paper are a lower bound (achievability result) and an upper bound (converse result) on $C_{W G I}$ for arbitrary $\underline{r}$ and sufficiently small $r_{s}$. To describe the main results, we need to introduce several parameters. Let

$$
f(\omega)=\sum_{i=0}^{k} c_{i} e^{\sqrt{-1} i \omega}
$$

be the discrete Fourier transform of the sequence $\underline{c}$,

$$
\alpha:=\min _{\omega \in[0,2 \pi]}|f(\omega)|, \quad \beta:=\max _{\omega \in[0,2 \pi]}|f(\omega)|
$$

and

$$
\varrho:=\left\{\begin{array}{cc}
\frac{\alpha}{1+\alpha^{2} P} & \frac{1}{P} \leq \alpha^{2} \\
\frac{1}{2 \sqrt{P}} & \alpha^{2}<\frac{1}{P}<\beta^{2} . \\
\frac{\beta}{1+\beta^{2} P} & \beta^{2} \leq \frac{1}{P}
\end{array}\right.
$$

If there exists an (unique) index $0 \leq i_{0} \leq k$ such that

$$
\frac{1}{6} P r_{i_{0}}^{2}>1+\frac{1}{6} P \sum_{i \neq i_{0}} r_{i}^{2}
$$

then define

$$
\phi_{1}:=\min _{L \in \mathbb{N}} L\left(1+\frac{1}{6} P \sum_{i \neq i_{0}} r_{i}^{2}+\frac{\frac{1}{6} P r_{i_{0}}^{2}}{L^{2}}\right)
$$

If there is no such index $i_{0}$, let

$$
\phi_{1}:=1+\frac{1}{6} P\|\underline{r}\|_{2}^{2}
$$

Also, define

$$
\phi_{2}:=\frac{2}{\sqrt{3}}\|\underline{r}\|_{2} \varrho P
$$




$$
\phi_{3}:=\frac{1}{\ln 2} r_{s}\left(r_{s}+\beta\right) P, \quad \phi_{4}:=\min \left\{r_{s}\left(r_{s}+\beta+\varrho\right) P, \frac{r_{s}\left(r_{s}+2 \beta\right) P}{1+\alpha^{2} P}\right\}
$$

and

$$
\phi_{5}:=\frac{1}{1+r_{s}\left(r_{s}+\beta+\varrho\right) P} .
$$

Throughout the paper, we assume $r_{s}$ is small enough such that

$$
r_{s}\left(r_{s}+2 \beta\right)<\alpha^{2}
$$

This guarantees that $\phi_{4}<1$ regardless of $P$. We are ready to present a lower bound on $C_{W G I}$.

Theorem 1. $C_{W G I}$ is bounded from below as

$$
C_{W G I} \geq C_{W G I}^{(\mathrm{lb})}:=\left(C_{W G I, 0}(P)-\delta\right)^{+}
$$

where

$$
\delta:=\log \phi_{1}+\min \left\{\phi_{3},-\frac{1}{2} \log \left(1-\phi_{4}\right)\right\}+\frac{1}{2 \ln 2}\left(1-\left(1-\phi_{2}\right)^{+} \phi_{5}\right) .
$$

Proof. The proof is detailed in Section V.

The lower bound in (28) is achieved by a joint-typicality decoder that is tuned to a set of candidates for the random channel matrix $\boldsymbol{H}$. This set forms a net that covers the range (ambiguity region) of $\boldsymbol{H}$. The optimization in (22) over the integer $L \geq 1$ is a result of adjusting the resolution of this net in order to achieve the largest possible rate. The choice $L=1$ simply means that the joint-typicality decoder is tuned to the single matrix $\mathbb{E}[\boldsymbol{H}]$. Throughout the paper, we denote the optimal choice of $L$ by $L_{\text {opt }}$.

Next, we provide an upper bound on $C_{W G I}$ :

Theorem 2. Let $k^{\prime}$ be the number of indices $0 \leq i \leq k$ such that $r_{i}>0$. Define

$$
\kappa:=\max \left\{\log \|\underline{r}\|_{\infty}, \frac{1}{2} \log k^{\prime}+\frac{1}{k^{\prime}} \sum_{i: r_{i}>0} \log r_{i}\right\} .
$$

Then $C_{W G I}$ is bounded from above as

$$
\begin{aligned}
C_{W G I} \leq C_{W G I}^{(\mathrm{ub})}:=\frac{1}{2} \log & \left(1+P\|\underline{r}\|_{2}^{2} / 3\right)+C_{W G I, 0}\left(\frac{P}{1+P\|\underline{r}\|_{2}^{2} / 3}\right) \\
& -\left(\frac{1}{2} \log \frac{P}{\pi e}+\kappa-\frac{\gamma_{e}}{2 \ln 2}\right)^{+},
\end{aligned}
$$


where the function $C_{W G I, 0}(\cdot)$ is given in (17) and $\gamma_{e} \approx 0.577$ is the Euler-Mascheroni constant.

Proof. See Appendix B.

Both $C_{W G I}^{(\mathrm{lb})}$ and $C_{W G I}^{(\mathrm{ub})}$ simplify to $C_{W G I, 0}(P)$ when $r_{0}=r_{1}=\cdots=r_{k}=0$.

In the special case where there is $r>0$ such that $r_{i}=r$ for every $0 \leq i \leq k$, we find $\kappa=\log r+\frac{1}{2} \log k$. The upper bound in Theorem 2 can be improved as

$$
\begin{aligned}
C_{W G I} \leq \frac{1}{2} \log & \left(1+k \operatorname{Pr}^{2} / 3\right)+C_{W G I, 0}\left(\frac{P}{1+k \operatorname{Pr}^{2} / 3}\right) \\
& -\left(\frac{1}{2} \log \frac{P}{\pi e}+\log r+\frac{1}{2} \log k+\frac{1}{2} \mathbb{E}\left[\log \chi_{k+1}^{2}\right]+\frac{1}{2}\right)^{+},
\end{aligned}
$$

where $\chi_{k+1}^{2}$ is a chi-square random variable with $k+1$ degrees of freedom. This is also verified at the end of Appendix B.

\section{SOME EXAMPLES}

In this section, we consider two examples in order to compare the lower and upper bounds provided in Theorem 1 and Theorem 2.

Example 1- Let us study the bounds $C_{W G I}^{(\mathrm{ub})}$ and $C_{W G I}^{(\mathrm{lb})}$ in the asymptote of large $P$. The upper bound in (31) does not scale with $P$. It saturates at

$$
\begin{aligned}
\lim _{P \rightarrow \infty} C_{W G I}^{(\mathrm{ub})} & =\frac{1}{2} \log \frac{\|\underline{r}\|_{2}^{2}}{3}+C_{W G I, 0}\left(\frac{3}{\|\underline{r}\|_{2}^{2}}\right)-\kappa+\frac{1}{2} \log (\pi e)+\frac{\gamma_{e}}{2 \ln 2} \\
& =\frac{1}{4 \pi} \int_{0}^{2 \pi} \log \left(\frac{\|\underline{r}\|_{2}^{2}}{3}+\left|\sum_{i=0}^{k} c_{i} e^{\sqrt{-1} i \omega}\right|^{2}\right) d \omega-\kappa+\frac{1}{2} \log (\pi e)+\frac{\gamma_{e}}{2 \ln 2}
\end{aligned}
$$

The lower bound in (28) vanishes for large values of $P$ unless there exists a unique index $0 \leq i \leq k$ such that $r_{i}>0$, i.e., only one of the taps is varying randomly from time slot to time slot. Denote this index (if it exists) by $i^{*}$ and let $r=r_{i^{*}}$. Then one can easily check that for large enough values of $P$,

$$
\phi_{1}=\min _{L \geq 1} L\left(1+\frac{\frac{1}{6} P r^{2}}{L^{2}}\right) \approx 2\left(\frac{1}{6} P r^{2}\right)^{1 / 2},
$$

where the optimum choice of $L$ is $L_{o p t}=\left[\left(\frac{1}{6} P r^{2}\right)^{1 / 2}\right]$ and ${ }^{1}$

$$
\delta \approx \log \phi_{1}-\frac{1}{2} \log \left(1-\frac{r(r+2 \beta)}{\alpha^{2}}\right)+\frac{1}{2 \ln 2}
$$

${ }^{1}$ When $P$ is large such that $\phi_{2}>1$, the term $\left(1-\phi_{2}\right)^{+} \phi_{5}$ disappears. Moreover, $\min \left\{\phi_{3},-\frac{1}{2} \log \left(1-\phi_{4}\right)\right\} \approx \frac{1}{2} \log \left(1-\frac{r(r+2 \beta)}{\alpha^{2}}\right)$. 


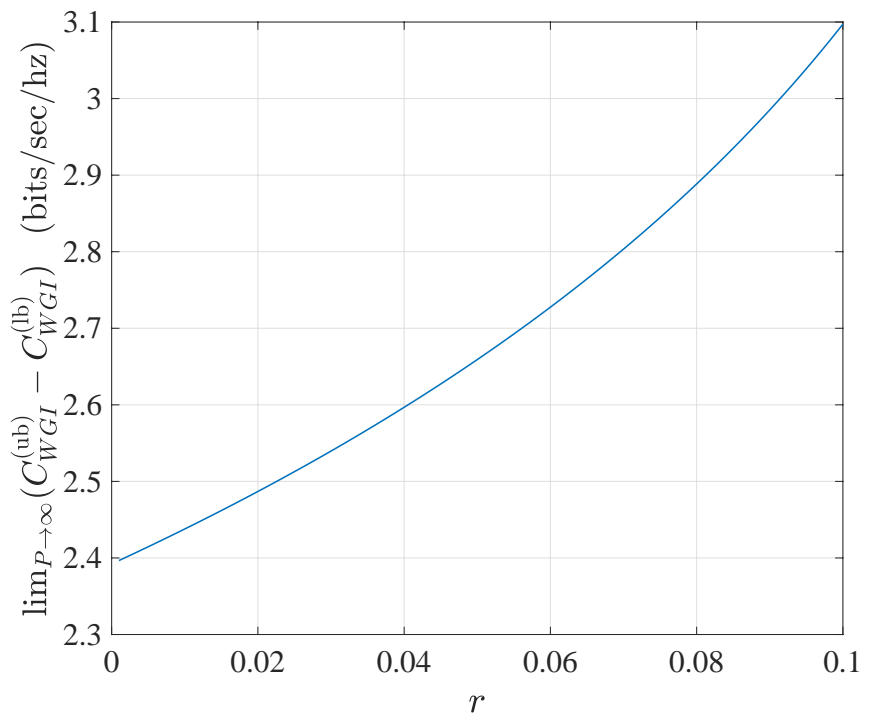

Fig. 1. Plot of $\lim _{P \rightarrow \infty}\left(C_{W G I}^{(\mathrm{ub})}-C_{W G I}^{(\mathrm{lb})}\right)$ in terms of $r$ in an ISI channel with $k=2, \underline{c}=(1,0.4,0.1)$ and $\underline{r}=(0,0, r)$.

This leads to

$$
\begin{aligned}
\lim _{P \rightarrow \infty} C_{W G I}^{(\mathrm{lb})}=\frac{1}{2 \pi} \int_{0}^{2 \pi} \log \mid & \sum_{i=0}^{k} c_{i} e^{\sqrt{-1} i \omega} \mid d \omega \\
& \quad \log r+\frac{1}{2} \log \left(1-\frac{r(r+2 \beta)}{\alpha^{2}}\right)-\log \frac{2}{\sqrt{6}}-\frac{1}{2 \ln 2} .
\end{aligned}
$$

Fig. 1 depicts the limiting difference $\lim _{P \rightarrow \infty}\left(C_{W G I}^{(\mathrm{ub})}-C_{W G I}^{(\mathrm{lb})}\right)$ in terms of $r$ in an ISI channel with $k=2$, $c_{0}=1, c_{1}=0.4, c_{2}=0.1$ and $r_{0}=0, r_{1}=0, r_{2}=r$.

If $r_{i}>0$ for more than one choice of $0 \leq i \leq k$, the term $\phi_{1}$ no longer scales like $P^{1 / 2}$. It scales like $P$ and hence, $\left(C_{W G I, 0}(P)-\delta\right)^{+}$will be eventually zero.

Example 2- Here we compare the bounds for finite values of $P$. Panel (a) in Fig. 2 presents plots of $C_{W G I}^{(\mathrm{ub})}$ and $C_{W G I}^{(\mathrm{lb})}$ in terms of $P$ (in $\mathrm{dBW}$ ) over an ISI channel with $k=3, \underline{c}=(1,0.1,0.1,0.1)$ and $\underline{r}=(0.01,0.05,0.01,0)$. The lower bound is plotted separately for $L=L_{o p t}$ and $L=1$. In both cases, the lower bound will eventually vanish as there are more than one index $i$ such that $r_{i}>0$. Panel (b) in Fig. 2 presents plots of $C_{W G I}^{(\mathrm{ub})}$ and $C_{W G I}^{(\mathrm{lb})}$ in terms of $P$ (in $\mathrm{dBW}$ ) over an ISI channel with $k=3$, $\underline{c}=(1,0.1,0.1,0.1)$ and $\underline{r}=(0,0,0.01,0)$. Since only one tap is random $\left(r_{2}>0\right)$, the lower bound with $L=L_{\text {opt }}$ will eventually saturate at a positive value as promised in the previous example. 


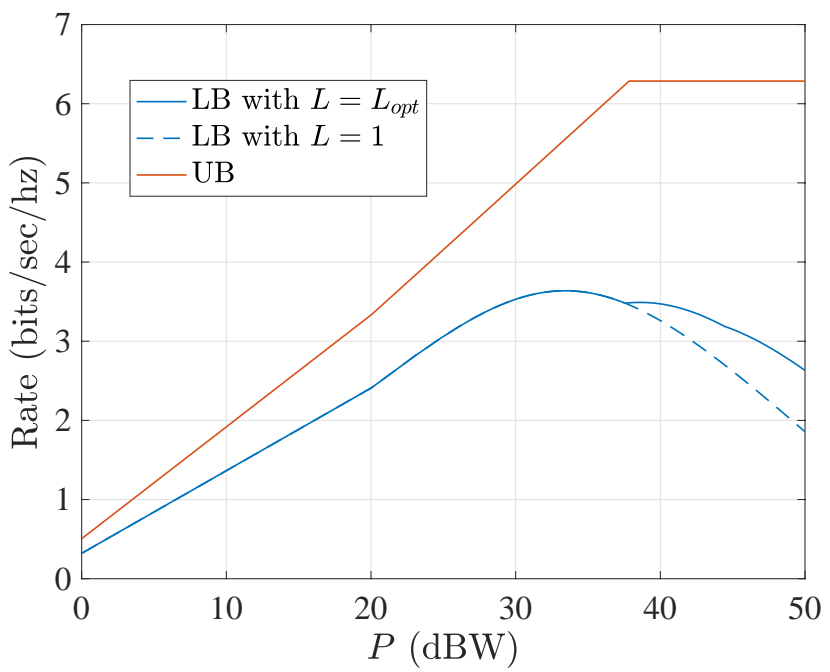

(a)

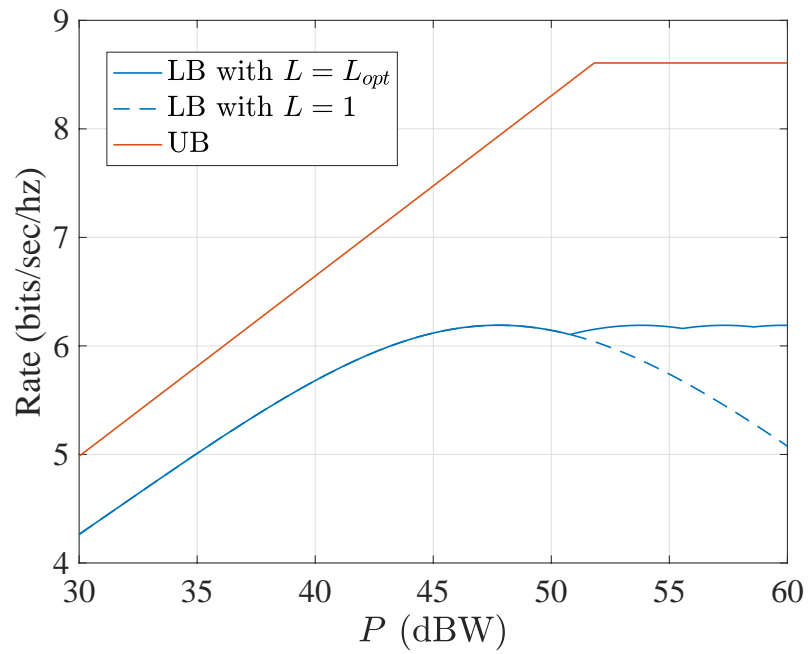

(b)

Fig. 2. Panel (a) presents plots of upper and lower bounds on $C_{W G I}$ in terms of $P(\mathrm{dBW})$ in an ISI channel with $k=3, \underline{c}=(1,0.1,0.1,0.1)$ and $\underline{r}=(0.01,0.05,0.01,0)$. Panel (b) presents the same plots in an ISI channel with $k=3, \underline{c}=(1,0.1,0.1,0.1)$ and $\underline{r}=(0,0,0.01,0)$.

\section{The Decoder StRucture}

The proposed decoder is quite simple and applies joint-typicality decoding over a net that covers the ambiguity region for the random channel matrix $\boldsymbol{H}$. Let $\mathcal{H}$ be the range of $\boldsymbol{H}$, i.e., $\mathcal{H}$ is the set of all $m \times n$ matrices for which $\left|H_{i, j}-c_{i-j}\right| \leq r_{i-j}$ for all $i, j$ such that $0 \leq i-j \leq k$ and $H_{i, j}=0$ otherwise.

Let $L_{0}, L_{1}, \cdots, L_{k} \geq 1$ be integers to be determined and define

$$
\tau_{0}=\frac{2 r_{0}}{L_{0}}, \tau_{1}=\frac{2 r_{1}}{L_{1}}, \cdots, \tau_{k}=\frac{2 r_{k}}{L_{k}}
$$

and

$$
\underline{\tau}:=\left[\begin{array}{llll}
\tau_{0} & \tau_{1} & \cdots & \tau_{k}
\end{array}\right]^{T} .
$$

Let $\mathcal{N}$ be a $\tau$-net over $\mathcal{H}$. By definition, $\mathcal{N}$ consists of $m \times n$ matrices $N$ that satisfy these two conditions:

(i) $N_{i, j}=0$ for $i-j<0$ or $i-j>k$.

(ii) For every $H \in \mathcal{H}$, there exists a matrix $N \in \mathcal{N}$ such that $\left|H_{i, j}-N_{i, j}\right| \leq \frac{1}{2} \tau_{i-j}$ for every $i, j$ such that $0 \leq i-j \leq k$.

It is clear that

$$
|\mathcal{N}|=\left(L_{0} L_{1} \cdots L_{k}\right)^{n} .
$$

Denote the codewords by the independent random vectors $\underline{\boldsymbol{x}}(i) \sim \mathrm{N}\left(\underline{0}_{n}, P I_{n}\right)$ for $i=1,2, \cdots, 2^{n R}$. 
Recall the definition of Gaussian typical sets in (5). The joint-typicality decoder looks for the unique index $i=1, \cdots, 2^{n R}$ for which there exists $N \in \mathcal{N}$ such that

$$
\underline{\boldsymbol{x}}(i) \in \mathcal{T}_{\epsilon}^{(n)}\left(P I_{n}\right), \underline{\boldsymbol{w}}(i) \in \mathcal{T}_{\eta}^{(m+n)}\left(\Sigma_{N}\right),
$$

where $\epsilon, \eta>0$ are constants and $\underline{\boldsymbol{w}}(i)$ and $\Sigma_{N}$ are defined by

$$
\underline{\boldsymbol{w}}(i):=\left[\begin{array}{ll}
\underline{\boldsymbol{x}}^{T}(i) & \underline{\boldsymbol{y}}^{T}
\end{array}\right]^{T}, \quad \Sigma_{N}:=\left[\begin{array}{cc}
P I_{n} & P N^{T} \\
P N & I_{m}+P N N^{T}
\end{array}\right] .
$$

The matrix $\Sigma_{N}$ is the covariance matrix for a vector $\left[\underline{\widetilde{x}}^{T} \underline{\widetilde{\boldsymbol{y}}}^{T}\right]^{T}$ where $\underline{\tilde{\boldsymbol{y}}}=N \underline{\widetilde{\boldsymbol{x}}}+\underline{\widetilde{\boldsymbol{z}}}$ and $\underline{\tilde{\boldsymbol{x}}}$ and $\underline{\tilde{z}}$ are independent $\mathrm{N}\left(\underline{0}_{n}, P I_{n}\right)$ and $\mathrm{N}\left(\underline{0}_{m}, I_{m}\right)$ random vectors, respectively. To construct the set $\mathcal{T}_{\eta}^{(m+n)}\left(\Sigma_{N}\right)$, one needs to compute $\Sigma_{N}^{-1}$. It is easy to see that $\Sigma_{N}^{-1}$ admits a closed form given by

$$
\Sigma_{N}^{-1}=\left[\begin{array}{cc}
\frac{1}{P} I_{n}+N^{T} N & -N^{T} \\
-N & I_{m}
\end{array}\right]
$$

It is also useful to note that ${ }^{2}$

$$
\operatorname{det}\left(\Sigma_{N}\right)=P^{n}
$$

regardless of the choice of $N \in \mathcal{N}$.

\section{ERROR ANALYSIS}

As usual, two types of error are distinguished:

(i) The transmitted codeword does not satisfy the decoding rule in (40). We refer to this as the type I error and denote it by $\mathcal{E}^{(I)}$.

(ii) A codeword different from the transmitted codeword satisfies (40). We refer to this as the type II error and denote it by $\mathcal{E}^{(I I)}$.

Then

$$
\left\langle p_{e}(\mathcal{C})\right\rangle_{\mathscr{C}} \leq \operatorname{Pr}\left(\mathcal{E}^{(I)}\right)+\operatorname{Pr}\left(\mathcal{E}^{(I I)}\right)
$$

In the following we examine the two terms on the right side of (44) separately.

\footnotetext{
${ }^{2}$ This follows by the Schur's complement formula for calculating the determinant of partitioned matrices [18].
} 
A. The probability of the type I error

Without loss of generality, assume $\underline{\boldsymbol{x}}(1)$ is the transmitted codeword, i.e., $\underline{\boldsymbol{y}}=\boldsymbol{H} \underline{\boldsymbol{x}}(1)+\underline{\boldsymbol{z}}$. Then

$$
\operatorname{Pr}\left(\mathcal{E}^{(I)}\right) \leq \operatorname{Pr}\left(\underline{\boldsymbol{x}}(1) \notin \mathcal{T}_{\epsilon}^{(n)}\left(P I_{n}\right)\right)+\operatorname{Pr}\left(\forall N \in \mathcal{N}, \underline{\boldsymbol{w}}(1) \notin \mathcal{T}_{\eta}^{(m+n)}\left(\Sigma_{N}\right)\right),
$$

where $\underline{\boldsymbol{w}}(1)$ and $\Sigma_{N}$ are given in (41). For every $\epsilon>0$, the first term on the right side of (45) tends to zero when $n$ grows due to the weak law of large numbers. The second term on the right side (45) is studied in the next proposition:

Proposition 1. Assume

$$
\eta>\eta_{n}:=\frac{n}{12(m+n)} P \sum_{l=0}^{k} \tau_{l}^{2} .
$$

Then

$$
\operatorname{Pr}\left(\forall N \in \mathcal{N}, \underline{\boldsymbol{w}}(1) \notin \mathcal{T}_{\eta}^{(m+n)}\left(\Sigma_{N}\right)\right) \leq C /\left((m+n)\left(\eta-\eta_{n}\right)^{2}\right),
$$

where

$$
C:=2\left(r_{s}^{2} P+r_{s} \sqrt{P}+1\right)^{2}+\frac{1}{360} P^{2} \sum_{l=0}^{k} \tau_{l}^{4}
$$

is a constant.

Proof. Let us write

$$
\underline{\boldsymbol{w}}(1)=\boldsymbol{A} \underline{\boldsymbol{\nu}},
$$

where $\boldsymbol{A}$ and $\underline{\boldsymbol{\nu}} \sim \mathrm{N}\left(\underline{0}_{m}, I_{m}\right)$ are given by

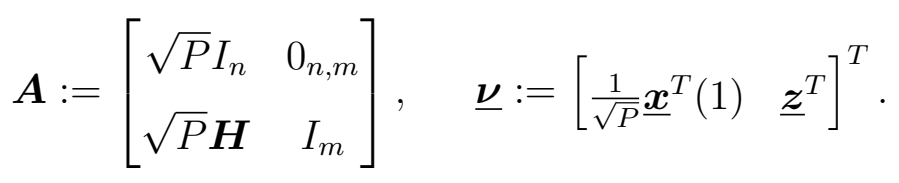

Then

$$
\underline{\boldsymbol{w}}^{T}(1) \Sigma_{N}^{-1} \underline{\boldsymbol{w}}(1)=\underline{\boldsymbol{\nu}}^{T} \boldsymbol{\Phi} \underline{\boldsymbol{\nu}}
$$


where $\Phi$ is defined by

$$
\boldsymbol{\Phi}:=\boldsymbol{A}^{T} \Sigma_{N}^{-1} \boldsymbol{A}
$$

Replacing the expression for $\Sigma_{N}^{-1}$ in (42), we find that

$$
\boldsymbol{\Phi}=\left[\begin{array}{cc}
I_{n}+P \boldsymbol{E}^{T} \boldsymbol{E} & \sqrt{P} \boldsymbol{E}^{T} \\
\sqrt{P} \boldsymbol{E} & I_{m}
\end{array}\right]
$$

where the "error matrix" $\boldsymbol{E}$ is defined by

$$
\boldsymbol{E}:=\boldsymbol{H}-N
$$

i.e., $\boldsymbol{E}$ is the difference between the actual channel matrix $\boldsymbol{H}$ and the matrix $N \in \mathcal{N}$. Define the ball $B_{\infty}(N, \underline{\tau})$ to be the set of all $m \times n$ matrices $H$ such that $\left|H_{i, j}-N_{i, j}\right| \leq \frac{1}{2} \tau_{i-j}$ for all $i, j$ such that $0 \leq i-j \leq k$. We write

$$
\begin{aligned}
\operatorname{Pr}\left(\forall N \in \mathcal{N}, \underline{\boldsymbol{w}}(1) \notin \mathcal{T}_{\eta}^{(m+n)}\left(\Sigma_{N}\right)\right) \\
\quad=\sum_{N^{\prime} \in \mathcal{N}} \operatorname{Pr}\left(\forall N \in \mathcal{N}, \underline{\boldsymbol{w}}(1) \notin \mathcal{T}_{\eta}^{(m+n)}\left(\Sigma_{N}\right) \mid \boldsymbol{H} \in B_{\infty}\left(N^{\prime}, \underline{\tau}\right)\right) \operatorname{Pr}\left(\boldsymbol{H} \in B_{\infty}\left(N^{\prime}, \underline{\tau}\right)\right), \\
\leq \sum_{N^{\prime} \in \mathcal{N}} \operatorname{Pr}\left(\underline{\boldsymbol{w}}(1) \notin \mathcal{T}_{\eta}^{(m+n)}\left(\Sigma_{N^{\prime}}\right) \mid \boldsymbol{H} \in B_{\infty}\left(N^{\prime}, \underline{\tau}\right)\right) \operatorname{Pr}\left(\boldsymbol{H} \in B_{\infty}\left(N^{\prime}, \underline{\tau}\right)\right),
\end{aligned}
$$

where we have used the fact that $\mathcal{N}$ is a $\underline{\tau}$-net for the range of $\boldsymbol{H}$. It is easy to see that the conditional probability law of the random matrix $\boldsymbol{H}$ given the condition $\boldsymbol{H} \in B_{\infty}\left(N^{\prime}, \underline{\tau}\right)$ is equal to the probability law of a random matrix that we denote by $\boldsymbol{H}_{\tau}$ for which $\left[\boldsymbol{H}_{\tau}\right]_{i, j}=0$ for $i-j<0$ or $i-j>k$ and $\left[\boldsymbol{H}_{\tau}\right]_{i, j}-\left[N^{\prime}\right]_{i, j}$ for $0 \leq i-j \leq k$ are independent uniform random variables on the interval $\left[-\frac{1}{2} \tau_{i-j}, \frac{1}{2} \tau_{i-j}\right]$. Let us define

$$
\boldsymbol{\Phi}_{\tau}:=\left[\begin{array}{cc}
I_{n}+P \boldsymbol{E}_{\tau}^{T} \boldsymbol{E}_{\tau} & \sqrt{P} \boldsymbol{E}_{\tau}^{T} \\
\sqrt{P} \boldsymbol{E}_{\tau} & I_{m}
\end{array}\right]
$$

where

$$
\boldsymbol{E}_{\tau}:=\boldsymbol{H}_{\tau}-N^{\prime}
$$


Then

$$
\begin{aligned}
& \operatorname{Pr}\left(\underline{\boldsymbol{w}}(1) \notin \mathcal{T}_{\eta}^{(m+n)}\left(\Sigma_{N^{\prime}}\right) \mid \boldsymbol{H} \in B_{\infty}\left(N^{\prime}, \underline{\tau}\right)\right) \\
& \quad=\operatorname{Pr}\left(\left|\underline{\boldsymbol{\nu}}^{T} \boldsymbol{\Phi} \underline{\boldsymbol{\nu}}-(m+n)\right| \geq(m+n) \eta \mid \boldsymbol{H} \in B_{\infty}\left(N^{\prime}, \underline{\tau}\right)\right) \\
& \quad=\operatorname{Pr}\left(\left|\underline{\boldsymbol{\nu}}^{T} \boldsymbol{\Phi}_{\tau} \underline{\boldsymbol{\nu}}-(m+n)\right| \geq(m+n) \eta\right),
\end{aligned}
$$

where the first step is due to (51) and the definition of $\mathcal{T}_{\eta}^{(m+n)}\left(\Sigma_{N^{\prime}}\right)$ and the second step follows the independence of $\underline{\nu}$ and $\boldsymbol{H}$ and definition of $\Phi_{\tau}$. We continue to further bound the term on the right side of (58). We have

$$
\begin{aligned}
\operatorname{Pr}\left(\left|\underline{\boldsymbol{\nu}}^{T} \boldsymbol{\Phi}_{\tau} \underline{\boldsymbol{\nu}}-(m+n)\right| \geq(m+n) \eta\right) & (m) \\
& \stackrel{(a)}{\leq} \operatorname{Pr}\left(\left|\underline{\boldsymbol{\nu}}^{T} \boldsymbol{\Phi}_{\tau} \underline{\boldsymbol{\nu}}-\mathbb{E}\left[\underline{\boldsymbol{\nu}}^{T} \boldsymbol{\Phi}_{\tau} \underline{\boldsymbol{\nu}}\right]\right|+\left|\mathbb{E}\left[\underline{\boldsymbol{\nu}}^{T} \boldsymbol{\Phi}_{\tau} \underline{\boldsymbol{\nu}}\right]-(m+n)\right| \geq(m+n) \eta\right) \\
& =\operatorname{Pr}\left(\left|\underline{\boldsymbol{\nu}}^{T} \boldsymbol{\Phi}_{\tau} \underline{\boldsymbol{\nu}}-\mathbb{E}\left[\underline{\boldsymbol{\nu}}^{T} \boldsymbol{\Phi}_{\tau} \underline{\boldsymbol{\nu}}\right]\right| \geq(m+n)\left(\eta-\left|\mathbb{E}\left[\underline{\boldsymbol{\nu}}^{T} \boldsymbol{\Phi}_{\tau} \underline{\boldsymbol{\nu}}\right] /(m+n)-1\right|\right)\right) \\
& \stackrel{(b)}{=} \operatorname{Pr}\left(\left|\underline{\boldsymbol{\nu}}^{T} \boldsymbol{\Phi}_{\tau} \underline{\boldsymbol{\nu}}-\mathbb{E}\left[\underline{\boldsymbol{\nu}}^{T} \boldsymbol{\Phi}_{\tau} \underline{\boldsymbol{\nu}}\right]\right| \geq(m+n)\left(\eta-P \mathbb{E}\left[\left\|\boldsymbol{E}_{\tau}\right\|_{2}^{2}\right] /(m+n)\right)\right),
\end{aligned}
$$

where $(a)$ follows by adding and subtracting $\mathbb{E}\left[\underline{\boldsymbol{\nu}}^{T} \boldsymbol{\Phi}_{\tau} \underline{\boldsymbol{\nu}}\right]$ and applying the triangle inequality and $(b)$ is due to

$$
\mathbb{E}\left[\underline{\boldsymbol{\nu}}^{T} \boldsymbol{\Phi}_{\tau} \underline{\boldsymbol{\nu}}\right]=\mathbb{E}\left[\operatorname{tr}\left(\boldsymbol{\Phi}_{\tau}\right)\right]=m+n+P \mathbb{E}\left[\left\|\boldsymbol{E}_{\tau}\right\|_{2}^{2}\right],
$$

which in turn follows from the independence of $\Phi_{\tau}$ and $\underline{\nu}$. But,

$$
\mathbb{E}\left[\left\|\boldsymbol{E}_{\tau}\right\|_{2}^{2}\right]=\sum_{i, j: 0 \leq i-j \leq k} \mathbb{E}\left[\left[\boldsymbol{E}_{\tau}\right]_{i, j}^{2}\right]=\frac{1}{12} n \sum_{l=0}^{k} \tau_{l}^{2},
$$

due to $\left[\boldsymbol{E}_{\tau}\right]_{i, j}$ being uniformly distributed over $\left[-\frac{1}{2} \tau_{i-j}, \frac{1}{2} \tau_{i-j}\right]$ for every $i, j$ such that $0 \leq i-j \leq k$. Putting (58), (59) and (61) together,

$$
\begin{aligned}
& \operatorname{Pr}\left(\underline{\boldsymbol{w}}(1) \notin \mathcal{T}_{\eta}^{(m+n)}\left(\Sigma_{N^{\prime}}\right) \mid \boldsymbol{H} \in B\left(N^{\prime}, \underline{\tau}\right)\right) \\
& \leq \operatorname{Pr}\left(\left|\underline{\boldsymbol{\nu}}^{T} \boldsymbol{\Phi}_{\tau} \underline{\boldsymbol{\nu}}-\mathbb{E}\left[\underline{\boldsymbol{\nu}}^{T} \boldsymbol{\Phi}_{\tau} \underline{\boldsymbol{\nu}}\right]\right| \geq(m+n)\left(\eta-\eta_{n}\right)\right) \\
& \leq \frac{\operatorname{Var}\left(\underline{\boldsymbol{\nu}}^{T} \boldsymbol{\Phi}_{\tau} \underline{\boldsymbol{\nu}}\right)}{(m+n)^{2}\left(\eta-\eta_{n}\right)^{2}}
\end{aligned}
$$

where $\eta_{n}$ is defined in (46) and the last step is due to $\eta>\eta_{n}$ and the Chebyshev's inequality. It is shown 
in Appendix $\mathrm{C}$ that

$$
\operatorname{Var}\left(\underline{\boldsymbol{\nu}}^{T} \boldsymbol{\Phi}_{\tau} \underline{\boldsymbol{\nu}}\right)=2 \mathbb{E}\left[\operatorname{tr}\left(\boldsymbol{\Phi}_{\tau}^{2}\right)\right]+\operatorname{Var}\left(\operatorname{tr}\left(\boldsymbol{\Phi}_{\tau}\right)\right)
$$

The term $\mathbb{E}\left[\operatorname{tr}\left(\boldsymbol{\Phi}_{\tau}^{2}\right)\right]$ can be bounded from above by

$$
\mathbb{E}\left[\operatorname{tr}\left(\boldsymbol{\Phi}_{\tau}^{2}\right)\right] \leq\left(r_{s}^{2} P+r_{s} \sqrt{P}+1\right)^{2}(m+n)
$$

The proof for (64) appears in Appendix D. Moreover,

$$
\begin{aligned}
\operatorname{Var}\left(\operatorname{tr}\left(\boldsymbol{\Phi}_{\tau}\right)\right) & =\operatorname{Var}\left(m+n+P\left\|\boldsymbol{E}_{\tau}\right\|_{2}^{2}\right) \\
& =P^{2} \operatorname{Var}\left(\left\|\boldsymbol{E}_{\tau}\right\|_{2}^{2}\right) \\
& =P^{2} \sum_{i, j: 0 \leq i-j \leq k} \operatorname{Var}\left(\left[\boldsymbol{E}_{\tau}\right]_{i-j}^{2}\right) \\
& =\frac{1}{180} n P^{2} \sum_{l=0}^{k} \tau_{l}^{4},
\end{aligned}
$$

where the last step is due to the fact that $\left[\boldsymbol{E}_{\tau}\right]_{i, j}$ for $0 \leq i-j \leq k$ is uniformly distributed on $\left[-\frac{1}{2} \tau_{i-j}, \frac{1}{2} \tau_{i-j}\right]$. Putting (55), (62), (63), (64) and (65) together, the proof is complete.

\section{B. The probability of the type II error}

As before, let $\underline{\boldsymbol{x}}(1)$ be the transmitted codeword. Define

$$
\Omega_{c}:=I_{m}+P H_{c} H_{c}^{T}
$$

where $H_{c}:=\mathbb{E}[\boldsymbol{H}]$ is an $m \times n$ matrix given by

$$
\left[H_{c}\right]_{i, j}=\left\{\begin{array}{cc}
c_{i-j} & 0 \leq i-j \leq k \\
0 & \text { otherwise }
\end{array} .\right.
$$


Note that if $r_{0}=r_{1}=\cdots=r_{k}=0$, the channel matrix $\boldsymbol{H}$ reduces to $H_{c}$. Let $\eta^{\prime}>0$ to be determined. The probability of the type II error can be bounded as

$$
\begin{aligned}
\operatorname{Pr}\left(\mathcal{E}^{(I I)}\right)= & \operatorname{Pr}\left(\exists i \neq 1, \quad \exists N \in \mathcal{N}, \underline{\boldsymbol{x}}(i) \in \mathcal{T}_{\epsilon}^{(n)}\left(P I_{n}\right), \underline{\boldsymbol{w}}(i) \in \mathcal{T}_{\eta}^{(m+n)}\left(\Sigma_{N}\right)\right) \\
\leq & \operatorname{Pr}\left(\underline{\boldsymbol{y}} \notin \mathcal{T}_{\eta^{\prime}}^{(m)}\left(\Omega_{c}\right)\right) \\
& \quad+\operatorname{Pr}\left(\exists i \neq 1, \quad \exists N \in \mathcal{N}, \underline{\boldsymbol{x}}(i) \in \mathcal{T}_{\epsilon}^{(n)}\left(P I_{n}\right), \underline{\boldsymbol{w}}(i) \in \mathcal{T}_{\eta}^{(m+n)}\left(\Sigma_{N}\right), \underline{\boldsymbol{y}} \in \mathcal{T}_{\eta^{\prime}}^{(m)}\left(\Omega_{c}\right)\right) \\
\leq & \operatorname{Pr}\left(\underline{\boldsymbol{y}} \notin \mathcal{T}_{\eta^{\prime}}^{(m)}\left(\Omega_{c}\right)\right) \\
& \quad+\sum_{i=2}^{2^{n R}} \sum_{N \in \mathcal{N}} \operatorname{Pr}\left(\underline{\boldsymbol{x}}(i) \in \mathcal{T}_{\epsilon}^{(n)}\left(P I_{n}\right), \underline{\boldsymbol{w}}(i) \in \mathcal{T}_{\eta}^{(m+n)}\left(\Sigma_{N}\right), \underline{\boldsymbol{y}} \in \mathcal{T}_{\eta^{\prime}}^{(m)}\left(\Omega_{c}\right)\right)
\end{aligned}
$$

where we have applied the union bound in the last step. The next proposition studies the first term on the right side of (68):

Proposition 2. Recall the definition of $\varrho$ in (20). Assume

$$
\eta^{\prime}>\eta_{n}^{\prime}:=\frac{2}{\sqrt{3}} \frac{n}{m}\|\underline{r}\|_{2} \varrho P .
$$

Then

$$
\operatorname{Pr}\left(\underline{\boldsymbol{y}} \notin \mathcal{T}_{\eta^{\prime}}^{(m)}\left(\Omega_{c}\right)\right) \leq C^{\prime} /\left(m\left(\eta^{\prime}-\eta_{n}^{\prime}\right)^{2}\right),
$$

where

$$
C^{\prime}:=2\left(\left(c_{s}+r_{s}\right)^{2} P+\left(c_{s}+r_{s}\right) \sqrt{P}+1\right)^{2}+\frac{P}{3}\|\underline{r}\|_{\infty}^{2}
$$

is a constant.

Proof. Let us write

$$
\underline{y}=B \underline{\nu},
$$

where

$$
\boldsymbol{B}:=\left[\sqrt{P} \boldsymbol{H} \quad I_{m}\right]
$$


and $\underline{\nu}$ is given in (50). Then

$$
\underline{\boldsymbol{y}}^{T} \Omega_{c}^{-1} \underline{\boldsymbol{y}}=\underline{\boldsymbol{\nu}}^{T} \boldsymbol{\Psi} \underline{\boldsymbol{\nu}}
$$

where $\Psi$ is defined by

$$
\boldsymbol{\Psi}:=\boldsymbol{B}^{T} \Omega_{c}^{-1} \boldsymbol{B}=\left[\begin{array}{cc}
P \boldsymbol{H}^{T} \Omega_{c}^{-1} \boldsymbol{H} & \sqrt{P} \boldsymbol{H}^{T} \Omega_{c}^{-1} \\
\sqrt{P} \Omega_{c}^{-1} \boldsymbol{H} & \Omega_{c}^{-1}
\end{array}\right] .
$$

Following similar lines of reasoning as in (59),

$$
\operatorname{Pr}\left(\underline{\boldsymbol{y}} \notin \mathcal{T}_{\eta^{\prime}}^{(m)}\left(\Omega_{c}\right)\right) \leq \operatorname{Pr}\left(\left|\underline{\boldsymbol{\nu}}^{T} \boldsymbol{\Psi} \underline{\boldsymbol{\nu}}-\mathbb{E}\left[\underline{\boldsymbol{\nu}}^{T} \boldsymbol{\Psi} \underline{\boldsymbol{\nu}}\right]\right| \geq m\left(\eta^{\prime}-\left|\mathbb{E}\left[\underline{\boldsymbol{\nu}}^{T} \boldsymbol{\Psi} \underline{\boldsymbol{\nu}}\right] / m-1\right|\right)\right) .
$$

Note that

$$
\begin{aligned}
\mathbb{E}\left[\underline{\boldsymbol{\nu}}^{T} \boldsymbol{\Psi} \underline{\boldsymbol{\nu}}\right] & \stackrel{(a)}{=} \mathbb{E}[\operatorname{tr}(\boldsymbol{\Psi})] \\
& \stackrel{(b)}{=} \mathbb{E}\left[\operatorname{tr}\left(\Omega_{c}^{-1}\left(I_{m}+P \boldsymbol{H} \boldsymbol{H}^{T}\right)\right)\right] \\
& \stackrel{(c)}{=} \mathbb{E}\left[\operatorname{tr}\left(\Omega_{c}^{-1}\left(\Omega_{c}+P\left(\boldsymbol{E}_{c} H_{c}^{T}+H_{c} \boldsymbol{E}_{c}^{T}\right)\right)\right]\right. \\
& =m+2 P \mathbb{E}\left[\operatorname{tr}\left(\Omega_{c}^{-1} H_{c} \boldsymbol{E}_{c}^{T}\right)\right],
\end{aligned}
$$

where (a) is due to independence of $\Psi$ and $\underline{\nu}$, (b) follows by the expression of $\Psi$ in (75) and the matrix $\boldsymbol{E}_{c}$ in $(c)$ is defined by

$$
\boldsymbol{E}_{c}:=\boldsymbol{H}-H_{c} .
$$

By (76) and (77),

$$
\operatorname{Pr}\left(\underline{\boldsymbol{y}} \notin \mathcal{T}_{\eta^{\prime}}^{(m)}\left(\Omega_{c}\right)\right) \leq \operatorname{Pr}\left(\left|\underline{\boldsymbol{\nu}}^{T} \boldsymbol{\Psi} \underline{\boldsymbol{\nu}}-\mathbb{E}\left[\underline{\boldsymbol{\nu}}^{T} \boldsymbol{\Psi} \underline{\boldsymbol{\nu}}\right]\right| \geq m\left(\eta^{\prime}-2 P\left|\mathbb{E}\left[\operatorname{tr}\left(\Omega_{c}^{-1} H_{c} \boldsymbol{E}_{c}^{T}\right)\right]\right| / m\right)\right)
$$


We have the thread of inequalities

$$
\begin{aligned}
\left|\mathbb{E}\left[\operatorname{tr}\left(\Omega_{c}^{-1} H_{c} \boldsymbol{E}^{T}\right)\right]\right| & \stackrel{(a)}{\leq} \mathbb{E}\left[\left|\operatorname{tr}\left(\Omega_{c}^{-1} H_{c} \boldsymbol{E}_{c}^{T}\right)\right|\right] \\
& \stackrel{(b)}{\leq} \mathbb{E}\left[\left\|\Omega_{c}^{-1} H_{c}\right\|_{2}\left\|\boldsymbol{E}_{c}\right\|_{2}\right] \\
& =\left\|\Omega_{c}^{-1} H_{c}\right\|_{2} \mathbb{E}\left[\left\|\boldsymbol{E}_{c}\right\|_{2}\right] \\
& \stackrel{(c)}{\leq}\left\|\Omega_{c}^{-1} H_{c}\right\|_{2}\left(\mathbb{E}\left[\left\|\boldsymbol{E}_{c}\right\|_{2}^{2}\right]\right)^{\frac{1}{2}} \\
& \stackrel{(d)}{=} \frac{1}{\sqrt{3}}\left\|\Omega_{c}^{-1} H_{c}\right\|_{2} \sqrt{n}\|\underline{r}\|_{2},
\end{aligned}
$$

where $(a)$ and $(c)$ are due to Jensen's inequality, $(b)$ is due to Cauchy-Schwarz inequality $\left|\operatorname{tr}\left(X Y^{T}\right)\right| \leq$ $\|X\|_{2}\|Y\|_{2}$ and $(d)$ is due to

$$
\mathbb{E}\left[\left\|\boldsymbol{E}_{c}\right\|_{2}^{2}\right]=\sum_{i, j: 0 \leq i-j \leq k} \mathbb{E}\left[\left(\boldsymbol{h}_{i, i-j}-c_{i-j}\right)^{2}\right]=\sum_{i, j: 0 \leq i-j \leq k} \frac{1}{3} r_{i-j}^{2}=\frac{1}{3} n\|\underline{r}\|_{2}^{2}
$$

The next lemma presents an upper bound on $\left\|\Omega_{c}^{-1} H_{c}\right\|_{2}$ :

Lemma 2. We have

$$
\left\|\Omega_{c}^{-1} H_{c}\right\|_{2} \leq \sqrt{n} \varrho
$$

where $\varrho$ is defined in (20).

Proof. See Appendix E.

By (80) and (82),

$$
\left|\mathbb{E}\left[\operatorname{tr}\left(\Omega_{c}^{-1} H_{c} \boldsymbol{E}^{T}\right)\right]\right| \leq \frac{1}{\sqrt{3}} n \varrho\|\underline{r}\|_{2}
$$

Now, (79) and (83) yield

$$
\operatorname{Pr}\left(\underline{\boldsymbol{y}} \notin \mathcal{T}_{\eta^{\prime}}^{(m)}\left(\Omega_{c}\right)\right) \leq \operatorname{Pr}\left(\left|\underline{\boldsymbol{\nu}}^{T} \boldsymbol{\Psi} \underline{\boldsymbol{\nu}}-\mathbb{E}\left[\underline{\boldsymbol{\nu}}^{T} \boldsymbol{\Psi} \underline{\boldsymbol{\nu}}\right]\right| \geq m\left(\eta^{\prime}-\eta_{n}^{\prime}\right)\right)
$$

where $\eta_{n}^{\prime}$ is defined in (69). Using the assumption $\eta^{\prime}>\eta_{n}^{\prime}$ and applying Chebyshev's inequality,

$$
\operatorname{Pr}\left(\underline{\boldsymbol{y}} \notin \mathcal{T}_{\eta^{\prime}}^{(m)}\left(\Omega_{c}\right)\right) \leq \frac{\operatorname{Var}\left(\underline{\boldsymbol{\nu}}^{T} \boldsymbol{\Psi} \underline{\boldsymbol{\nu}}\right)}{m^{2}\left(\eta^{\prime}-\eta_{n}^{\prime}\right)^{2}} .
$$


Following similar lines of reasoning that led to (63), we can write

$$
\operatorname{Var}\left(\underline{\boldsymbol{\nu}}^{T} \boldsymbol{\Psi} \underline{\boldsymbol{\nu}}\right)=2 \mathbb{E}\left[\operatorname{tr}\left(\boldsymbol{\Psi}^{2}\right)\right]+\operatorname{Var}(\operatorname{tr}(\boldsymbol{\Psi}))
$$

An upper bound on $\mathbb{E}\left[\operatorname{tr}\left(\Psi^{2}\right)\right]$ is given by

$$
\mathbb{E}\left[\operatorname{tr}\left(\Psi^{2}\right)\right] \leq\left(\left(c_{s}+r_{s}\right)^{2} P+\left(c_{s}+r_{s}\right) \sqrt{P}+1\right)^{2}(m+n)
$$

The proof is slightly different from that of (64). The details appear in Appendix F. Finally, we compute an upper bound on the second term on the right side of (86). Recall from (77) $\operatorname{that} \operatorname{tr}(\Psi)=m+$ $2 P \operatorname{tr}\left(\Omega_{c}^{-1} H_{c} \boldsymbol{E}_{c}^{T}\right)$. Then

$$
\begin{aligned}
\operatorname{Var}(\operatorname{tr}(\boldsymbol{\Psi})) & =4 P^{2} \operatorname{Var}\left(\operatorname{tr}\left(\Omega_{c}^{-1} H_{c} \boldsymbol{E}_{c}^{T}\right)\right) \\
& \left.=4 P^{2} \operatorname{Var}\left(\sum_{i, j}\left[\Omega_{c}^{-1} H_{c}\right]_{i, j}\left[\boldsymbol{E}_{c}\right]_{i, j}\right)\right) \\
& \left.\stackrel{(a)}{=} 4 P^{2} \operatorname{Var}\left(\sum_{i, j: 0 \leq i-j \leq k}\left[\Omega_{c}^{-1} H_{c}\right]_{i, j}\left[\boldsymbol{E}_{c}\right]_{i, j}\right)\right) \\
& \stackrel{(b)}{=} 4 P^{2} \sum_{i, j: 0 \leq i-j \leq k}\left[\Omega_{c}^{-1} H_{c}\right]_{i, j}^{2} \operatorname{Var}\left(\left[\boldsymbol{E}_{c}\right]_{i, j}\right) \\
& \stackrel{(c)}{=} \frac{4}{3} P^{2} \sum_{i, j: 0 \leq i-j \leq k}\left[\Omega_{c}^{-1} H_{c}\right]_{i, j}^{2} r_{i-j}^{2} \\
& \stackrel{(d)}{\leq} \frac{4}{3} P^{2}\|\underline{r}\|_{\infty}^{2}\left\|\Omega_{c}^{-1} H_{c}\right\|_{2}^{2} \\
& \stackrel{(e)}{\leq} \frac{1}{3} P\|\underline{r}\|_{\infty}^{2} n,
\end{aligned}
$$

where $(a)$ is due to $\left[\boldsymbol{E}_{c}\right]_{i, j}=0$ for $i-j<0$ or $i-j>k,(b)$ is due to $\left[\boldsymbol{E}_{c}\right]_{i, j}$ being independent for all pairs $(i, j)$ with $0 \leq i-j \leq k,(c)$ is due to $\left[\boldsymbol{E}_{c}\right]_{i, j}$ being uniformly distributed on $\left[-r_{i-j}, r_{i-j}\right],(d)$ follows by bounding $r_{i-j}^{2}$ and $\sum_{i, j: 0 \leq i-j \leq k}\left[\Omega_{c}^{-1} H_{c}\right]_{i, j}^{2}$ by $\|\underline{r}\|_{\infty}^{2}$ and $\sum_{i, j}\left[\Omega_{c}^{-1} H_{c}\right]_{i, j}^{2}=\left\|\Omega_{c}^{-1} H_{c}\right\|_{2}^{2}$, respectively, and finally $(e)$ is due to Lemma 2 and the fact that $\varrho \leq \frac{1}{2 \sqrt{P}}$. This completes the proof. 
Next, let us concentrate on the second term on the right side of (68). We write

$$
\begin{aligned}
\operatorname{Pr} & \left(\underline{\boldsymbol{x}}(i) \in \mathcal{T}_{\epsilon}^{(n)}\left(P I_{n}\right), \underline{\boldsymbol{w}}(i) \in \mathcal{T}_{\eta}^{(m+n)}\left(\Sigma_{N}\right), \underline{\boldsymbol{y}} \in \mathcal{T}_{\eta^{\prime}}^{(m)}\left(\Omega_{c}\right)\right) \\
& \stackrel{(a)}{=} \int_{\mathcal{T}_{\eta}^{(m+n)}\left(\Sigma_{N}\right) \cap\left(\mathcal{T}_{\epsilon}^{(n)}\left(P I_{n}\right) \times \mathcal{T}_{\eta^{\prime}}^{(m)}\left(\Omega_{c}\right)\right)} p_{\underline{\boldsymbol{x}}(i)}(\underline{x}) p_{\underline{\boldsymbol{y}}}(\underline{y}) \mathrm{d} \underline{x} \mathrm{~d} \underline{y} \\
& \stackrel{(b)}{\leq} 2^{-h_{\mathrm{G}}\left(P I_{n}\right)+\frac{1}{2 \ln 2} n \epsilon} \times \operatorname{Vol}\left(\mathcal{T}_{\eta}^{(m+n)}\left(\Sigma_{N}\right)\right) \sup _{\underline{y} \in \mathcal{T}_{\eta^{\prime}}^{(m)}\left(\Omega_{c}\right)} p_{\underline{\boldsymbol{y}}}(\underline{y}) \\
& \stackrel{(c)}{\leq} 2^{-h_{\mathrm{G}}\left(P I_{n}\right)+\frac{1}{2 \ln 2} n \epsilon} \times 2^{h_{\mathrm{G}}\left(\Sigma_{N}\right)+\frac{m+n}{2} \log (1+\eta)} \sup _{\underline{y} \in \mathcal{T}_{\eta^{\prime}}^{(m)}\left(\Omega_{c}\right)} p_{\underline{\boldsymbol{y}}}(\underline{y}) \\
& \stackrel{(d)}{\leq} 2^{\frac{m+n}{2} \log (1+\eta)+\frac{1}{2 \ln 2} n \epsilon}(2 \pi e)^{\frac{m}{2}} \sup _{\underline{y} \in \mathcal{T}_{\eta^{\prime}}^{(m)}\left(\Omega_{c}\right)} p_{\underline{\boldsymbol{y}}}(\underline{y}),
\end{aligned}
$$

where $(a)$ is due to independence of $\underline{\boldsymbol{x}}(i)$ and $\underline{\boldsymbol{y}}$ for $i \neq 1,(b)$ is due to $p_{\underline{\boldsymbol{x}}(i)}(\underline{x})=p_{\mathrm{G}}\left(\underline{x} ; P I_{n}\right) \leq$ $2^{-h_{\mathrm{G}}\left(P I_{n}\right)+\frac{1}{2 \ln 2} n \epsilon}$ for $\underline{x} \in \mathcal{T}_{\epsilon}^{(n)}\left(P I_{n}\right),(c)$ is due to Lemma 1 on the volume of typical sets and $(d)$ is due to

$$
h_{\mathrm{G}}\left(\Sigma_{N}\right)-h_{\mathrm{G}}\left(P I_{n}\right)=\frac{1}{2} \log \left((2 \pi e)^{m+n} \operatorname{det}\left(\Sigma_{N}\right)\right)-\frac{1}{2} \log (2 \pi e P)^{n}=\frac{m}{2} \log (2 \pi e)
$$

regardless of the choice of $N \in \mathcal{N}$ which in turn follows from (43). Note that $p_{\underline{y}}(\underline{y}) \neq p_{\mathrm{G}}\left(\underline{y} ; \Omega_{c}\right)$ and hence, we can not bound $p_{\underline{\boldsymbol{y}}}(\underline{y})$ for $\underline{y} \in \mathcal{T}_{\eta^{\prime}}^{(m)}\left(\Omega_{c}\right)$ in a similar fashion as we bounded $p_{\underline{x}}(i)(\underline{x})$ for $\underline{x} \in \mathcal{T}_{\epsilon}^{(n)}\left(P I_{n}\right)$ in step $(b)$ in (89). We proceed by presenting an upper bound on the supremum on the right side of (89). Recall we denoted the range of the random matrix $\boldsymbol{H}$ by $\mathcal{H}$. We write

$$
\begin{aligned}
p_{\underline{\boldsymbol{y}}}(\underline{y}) & =\int_{\mathcal{H}} p_{\underline{\boldsymbol{y}}}(\underline{y} \mid \boldsymbol{H}=H) d \mathcal{L}_{\boldsymbol{H}}(H) \\
& =\int_{\mathcal{H}} \frac{1}{(2 \pi)^{\frac{m}{2}}\left(\operatorname{det}\left(I_{m}+P H H^{T}\right)\right)^{\frac{1}{2}}} \exp \left(-\frac{1}{2} \underline{y}^{T}\left(I_{m}+P H H^{T}\right)^{-1} \underline{y}\right) d \mathcal{L}_{\boldsymbol{H}}(H) \\
& \leq(2 \pi)^{-\frac{m}{2}}\left(\inf _{H \in \mathcal{H}} \operatorname{det}\left(\Omega_{H}\right)\right)^{-\frac{1}{2}} \exp \left(-\frac{1}{2} \inf _{H \in \mathcal{H}} \underline{y}^{T} \Omega_{H}^{-1} \underline{y}\right)
\end{aligned}
$$

where $\mathcal{L}_{\boldsymbol{H}}$ is the probability law of $\boldsymbol{H}$ and we have defined

$$
\Omega_{H}:=I_{m}+P H H^{T}
$$

Then

$$
\sup _{\underline{y} \in \mathcal{T}_{\eta^{\prime}}^{(m)}\left(\Omega_{c}\right)} p_{\underline{\boldsymbol{y}}}(\underline{y}) \leq(2 \pi)^{-\frac{m}{2}}\left(\inf _{H \in \mathcal{H}} \operatorname{det}\left(\Omega_{H}\right)\right)^{-\frac{1}{2}} \exp \left(-\frac{1}{2} \inf _{H \in \mathcal{H}} \inf _{\underline{y} \in \mathcal{T}_{\eta^{\prime}}^{(m)}\left(\Omega_{c}\right)} \underline{y}^{T} \Omega_{H}^{-1} \underline{y}\right) .
$$

The next two lemmas provide lower bounds on the infimums on the right side of (93): 
Lemma 3. Recall $\phi_{3}$ and $\phi_{4}$ in (25). Then

$$
\inf _{H \in \mathcal{H}} \operatorname{det}\left(\Omega_{H}\right) \geq 2^{-2 m \phi_{3}} \operatorname{det}\left(\Omega_{c}\right)
$$

and

$$
\inf _{H \in \mathcal{H}} \operatorname{det}\left(\Omega_{H}\right) \geq\left(1-\phi_{4}\right)^{m} \operatorname{det}\left(\Omega_{c}\right)
$$

Proof. The proof for (94) uses a perturbation argument and the Mean Value Theorem. The proof for (95) relies in part on Weyl's inequality on perturbation of eigenvalues of a symmetric matrix. See Appendix G for the details.

Lemma 4. Recall $\phi_{5}$ in (26). Then

$$
\inf _{H \in \mathcal{H}} \inf _{\underline{y} \in \mathcal{T}_{\eta^{\prime}}^{(m)}\left(\Omega_{c}\right)} \underline{y}^{T} \Omega_{H}^{-1} \underline{y} \geq m\left(1-\eta^{\prime}\right)^{+} \phi_{5}
$$

Proof. The inner optimization is a quadratically constrained quadratic convex program. See Appendix $\mathrm{H}$ for the details.

By (93), (94), (95) and (96),

$$
\sup _{\underline{y} \in \mathcal{T}_{\eta^{\prime}}^{(m)}\left(\Omega_{c}\right)} p_{\boldsymbol{y}}(\underline{y}) \leq(2 \pi)^{-\frac{m}{2}} 2^{-\frac{1}{2} \log \operatorname{det}\left(\Omega_{c}\right)} 2^{m \min \left\{\phi_{3},-\frac{1}{2} \log \left(1-\phi_{4}\right)\right\}} \exp \left(-\frac{m}{2}\left(1-\eta^{\prime}\right)^{+} \phi_{5}\right),
$$

Putting (68), (70), (89) and (97) together, the probability of type II error is bounded by

$$
\operatorname{Pr}\left(\mathcal{E}^{(I I)}\right) \leq C^{\prime} /\left(m\left(\eta^{\prime}-\eta_{n}^{\prime}\right)^{2}\right)+2^{n\left(R-\frac{1}{2 n} \log \operatorname{det}\left(\Omega_{c}\right)+\delta_{n}\right)}
$$

where $\delta_{n}$ in the exponent is given by

$$
\begin{gathered}
\delta_{n}=\frac{1}{n} \log |\mathcal{N}|+\frac{m+n}{2 n} \log (1+\eta)+\frac{m}{n} \min \left\{\phi_{3},-\frac{1}{2} \log \left(1-\phi_{4}\right)\right\} \\
+\frac{m}{2 n \ln 2}\left(1-\left(1-\eta^{\prime}\right)^{+} \phi_{5}\right)+\frac{\epsilon}{2 \ln 2} .
\end{gathered}
$$

and we have the conditions $\eta^{\prime}>\eta_{n}^{\prime}$ and $\eta>\eta_{n}$. The right side of (98) vanishes as $n$ grows if the two inequalities

$$
R<\lim _{n \rightarrow \infty} \frac{1}{2 n} \log \operatorname{det}\left(\Omega_{c}\right)-\lim _{n \rightarrow \infty} \delta_{n}, \quad \eta^{\prime}>\lim _{n \rightarrow \infty} \eta_{n}^{\prime}=\phi_{2}
$$


hold and the right side of (47) vanishes as $n$ grows if

$$
\eta>\lim _{n \rightarrow \infty} \eta_{n}=\frac{1}{24} P \sum_{i=0}^{k} \tau_{i}^{2} .
$$

We can write

$$
\begin{aligned}
\lim _{n \rightarrow \infty} \frac{1}{2 n} \log \operatorname{det}\left(\Omega_{c}\right) & =\lim _{n \rightarrow \infty} \frac{1}{2 n} \log \operatorname{det}\left(I_{m}+P H_{c} H_{c}^{T}\right) \\
& =\lim _{n \rightarrow \infty} \frac{1}{2 n} \log \operatorname{det}\left(I_{n}+P H_{c}^{T} H_{c}\right) \\
& =C_{W G I, 0}(P),
\end{aligned}
$$

where the penultimate step is $\operatorname{due}$ to $\operatorname{det}\left(I_{m}+P H_{c} H_{c}^{T}\right)=\operatorname{det}\left(I_{n}+P H_{c}^{T} H_{c}\right)$ and the last step is due to Szegö's Theorem [19] after one recognizes that the matrix $H_{c}^{T} H_{c}$ is a banded Toeplitz matrix given by $\left[H_{c}^{T} H_{c}\right]_{i, j}=\left(\sum_{l=0}^{k-|i-j|} c_{l} c_{l+|i-j|}\right) \mathbb{1}_{|i-j| \leq k}$. Also,

$$
\begin{aligned}
\lim _{n \rightarrow \infty} \delta_{n}= & \log \left(L_{0} L_{1} \cdots L_{k}\right)+\log (1+\eta)+ \\
& \min \left\{\phi_{3},-\frac{1}{2} \log \left(1-\phi_{4}\right)\right\}+\frac{1}{2 \ln 2}\left(1-\left(1-\eta^{\prime}\right)^{+} \phi_{5}\right)+\frac{\epsilon}{2 \ln 2},
\end{aligned}
$$

where $\eta$ and $\eta^{\prime}$ satisfy $\eta>\frac{1}{24} P \sum_{i=0}^{k} \tau_{i}^{2}=\frac{1}{6} P \sum_{i=0}^{k} \frac{r_{i}^{2}}{L_{i}^{2}}$ and $\eta^{\prime}>\phi_{2}$. The smallest value for $\lim _{n \rightarrow \infty} \delta_{n}$ is achieved when $\eta$ and $\eta^{\prime}$ are replaced by $\frac{1}{6} P \sum_{i=0}^{k} \frac{r_{i}^{2}}{L_{i}^{2}}$ and $\phi_{2}$, respectively. Finally, we choose the integers $L_{0}, L_{1}, \cdots, L_{k}$ to minimize $\log \left(L_{0} L_{1} \cdots L_{k}\right)+\log \left(1+\frac{1}{6} P \sum_{i=0}^{k} \frac{r_{i}^{2}}{L_{i}^{2}}\right)$. Induction shows that the minimum is achieved when at least $k$ of the variables $L_{0}, L_{1}, \cdots, L_{k}$ are 1 . Without loss of generality, let $L_{1}=L_{2}=\cdots=L_{k}=1$. Then the objective function to be minimized is $L_{0}\left(1+\frac{1}{6} P \sum_{i=1}^{k} r_{i}^{2}+\frac{\frac{1}{6} \operatorname{Pr}_{0}^{2}}{L_{0}^{2}}\right)$. The minimum is achieved by $L_{0}=\max \left\{1,\left[\left(\frac{\frac{1}{6} P r_{0}^{2}}{1+\frac{1}{6} P \sum_{i=1}^{k} r_{i}^{2}}\right)^{1 / 2}\right]\right\}$.

\section{SUMMARY AND CONCLUDING REMARKS}

We studied a stochastic and time varying Gaussian ISI channel where $\boldsymbol{h}_{t, i}$, the $i^{\text {th }}$ tap during time slot $t$, was uniformly distributed over $\left[c_{i}-r_{i}, c_{i}+r_{i}\right]$. The array $\boldsymbol{h}_{t, i}$ was assumed to be independent along both indices $t$ and $i$. Moreover, this array was unknown to both ends of transmission. An upper bound and a lower bound were derived on $C_{W G I}$, i.e., the largest achievable rate that can be achieved by white Gaussian codewords.

It was shown that that the proposed upper bound saturates with the average transmission power $P$. As such, $C_{W G I}$ does not scale with $P$. The lower bound was achieved by a joint-typicality decoder tuned to 
a net of candidates for the random channel matrix $\boldsymbol{H}$. The net covered the range of $\boldsymbol{H}$ and its resolution was optimized to obtain the largest achievable rate. It was shown that if only one of the channel taps is changing randomly (e.g, the third tap in all times $t$ ), then the lower bound also saturates at a positive level as $P$ increases. Otherwise, the lower bound vanishes for sufficiently large $P$. It remains unclear if this behaviour is intrinsic to joint-typicality decoding or an error analysis conducted more carefully would show that the proposed decoder always achieves positive rates regardless of how many taps are changing randomly. One direction this may be examined is to see if an upper bound more sophisticated than the naive union bound may be employed in order to bound the probability of the type II error.

An interesting problem not addressed in this paper is to examine the robustness of universal decoders in [8], [9] and [10] to variations in the unknown channel taps as modelled in this paper. It is also curious if employing such decoders may lead us to a closed expression for or a tighter lower bound on $C_{W G I}$.

\section{AppendiX A; Proof OF LEMMA 1}

For $r>0$, positive integer $n$ and real positive-definite matrix $\Sigma$, consider the ellipsoid

$$
\mathcal{E}_{r}:=\left\{\underline{a} \in \mathbb{R}^{n}: \frac{1}{n} \underline{a}^{T} \Sigma^{-1} \underline{a}<r .\right\} .
$$

Let $\Sigma=U \Lambda U^{T}$ be the spectral decomposition for $\Sigma$ where $U$ is a real orthogonal matrix and $\Lambda$ is a diagonal matrix whose diagonal entries $\lambda_{1}, \cdots, \lambda_{N}$ are the eigenvalues of $\Sigma$. The set $U^{T} \mathcal{E}_{r}:=\left\{U^{T} \underline{a}: \underline{a} \in \mathcal{E}_{r}\right\}$ is an ellipsoid in standard from, i.e.,

$$
U^{T} \mathcal{E}_{r}=\left\{\underline{a} \in \mathbb{R}^{n}: \frac{1}{n} \sum_{i=1}^{n} \frac{a_{i}^{2}}{\lambda_{i}}<r\right\}
$$


The volume of the standard ellipsoid $\left\{\left(a_{1}, \cdots, a_{n}\right): \sum_{i=1}^{n} \frac{a_{i}^{2}}{c_{i}^{2}} \leq 1\right\}$ is $\frac{\pi^{\frac{n}{2}}}{\Gamma\left(\frac{n}{2}+1\right)} \prod_{i=1}^{n} c_{i}$ where $\Gamma(\cdot)$ is the Gamma function. Hence,

$$
\begin{aligned}
\operatorname{Vol}\left(\mathcal{E}_{r}\right) & \stackrel{(a)}{=} \operatorname{Vol}\left(U^{T} \mathcal{E}_{r}\right) \\
& =\frac{\pi^{\frac{n}{2}}}{\Gamma\left(\frac{n}{2}+1\right)} \prod_{i=1}^{n}\left(n r \lambda_{i}\right)^{\frac{1}{2}} \\
& \stackrel{(b)}{=} \frac{\pi^{\frac{n}{2}} n^{\frac{n}{2}}}{\Gamma\left(\frac{n}{2}+1\right)}(\operatorname{det}(\Sigma))^{\frac{1}{2}} r^{\frac{n}{2}} \\
& \stackrel{(c)}{\leq}(2 \pi e)^{\frac{n}{2}}(\operatorname{det}(\Sigma))^{\frac{1}{2}} r^{\frac{n}{2}} \\
& =2^{\frac{1}{2} \log \left((2 \pi e)^{n} \operatorname{det}(\Sigma)\right)+\frac{n}{2} \log r} \\
& =2^{h_{\mathrm{G}}(\Sigma)+\frac{n}{2} \log r},
\end{aligned}
$$

where $(a)$ is due to the fact that the map $\underline{a} \mapsto U^{T} \underline{a}$ is an isometry, $(b)$ is due to $\operatorname{det}(\Sigma)=\prod_{i=1}^{n} \lambda_{i}$ and (c) is due to the inequality $\Gamma(x+1) \geq x^{x} e^{-x}$ for any $x>0$ according to Corollary 1.2 in [17]. Finally,

$$
\operatorname{Vol}\left(\mathcal{T}_{\eta}^{(n)}(\Sigma)\right) \leq \operatorname{Vol}\left(\mathcal{E}_{1+\eta}\right) \leq 2^{h_{\mathrm{G}}(\Sigma)+\frac{n}{2} \log (1+\eta)}
$$

\section{APpendix B; Proof of TheOREM 2}

Let us fix a codebook $\mathcal{C}=\left\{\underline{x}(1), \cdots, \underline{x}\left(2^{n R}\right)\right\}$. Then the transmitted vector $\underline{\boldsymbol{x}}$ in (12) is uniformly distributed over $\mathcal{C}$. We write

$$
\begin{aligned}
n R & =H(\boldsymbol{W}) \\
& =H(\boldsymbol{W} \mid \underline{\boldsymbol{y}})+I(\boldsymbol{W} ; \underline{\boldsymbol{y}}) \\
& \leq 1+n p_{e}(\mathcal{C}) R+I(\underline{\boldsymbol{x}} ; \underline{\boldsymbol{y}}),
\end{aligned}
$$

where the last step is due to the Fano's inequality and the data processing inequality for mutual information. Averaging with respect to the codebook $\mathcal{C}$ over the Gaussian ensemble $\mathscr{C}$,

$$
n R \leq 1+n R\left\langle p_{e}(\mathcal{C})\right\rangle_{\mathscr{C}}+\langle I(\underline{\boldsymbol{x}} ; \underline{\boldsymbol{y}})\rangle_{\mathscr{C}}
$$

Let us write

$$
\langle I(\underline{\boldsymbol{x}} ; \underline{\boldsymbol{y}})\rangle_{\mathscr{C}}=\langle h(\underline{\boldsymbol{y}})\rangle_{\mathscr{C}}-\langle h(\underline{\boldsymbol{y}} \mid \underline{\boldsymbol{x}})\rangle_{\mathscr{C}}
$$

Next, we find an upper bound and a lower bound on the terms $\langle h(\underline{\boldsymbol{y}})\rangle_{\mathscr{C}}$ and $\langle h(\underline{\boldsymbol{y}} \mid \underline{\boldsymbol{x}})\rangle_{\mathscr{C}}$, respectively. 
A. Upper bound on $\langle h(\underline{\boldsymbol{y}})\rangle_{\mathscr{C}}$

Applying the maximum entropy lemma [20],

$$
h(\underline{\boldsymbol{y}}) \leq \frac{1}{2} \log \left((2 \pi e)^{m} \operatorname{det}(\operatorname{Cov}(\underline{\boldsymbol{y}}))\right)
$$

Define

$$
\underline{\mathrm{m}}(\mathcal{C}):=\mathbb{E}[\underline{\boldsymbol{x}}]=\frac{1}{2^{n R}} \sum_{i=1}^{2^{n R}} \underline{x}(i), \quad \mathrm{Q}(\mathcal{C}):=\mathbb{E}\left[\underline{\boldsymbol{x}} \underline{\boldsymbol{x}}^{T}\right]=\frac{1}{2^{n R}} \sum_{i=1}^{2^{n R}} \underline{x}(i) \underline{x}^{T}(i)
$$

A simple computation shows that

$$
\operatorname{Cov}(\underline{\boldsymbol{y}})=I_{m}+\mathbb{E}\left[\boldsymbol{H Q}(\mathcal{C}) \boldsymbol{H}^{T}\right]-\mathbb{E}[\boldsymbol{H}] \underline{\mathrm{m}}(\mathcal{C}) \underline{\mathrm{m}}^{T}(\mathcal{C}) \mathbb{E}\left[\boldsymbol{H}^{T}\right]
$$

By (111) and (113) and using the fact that $\log \operatorname{det}(\cdot)$ is nondecreasing along the cone of positivesemidefinite matrices,

$$
h(\underline{\boldsymbol{y}}) \leq \frac{1}{2} \log \left((2 \pi e)^{m} \operatorname{det}\left(I_{m}+\mathbb{E}\left[\boldsymbol{H Q}(\mathcal{C}) \boldsymbol{H}^{T}\right]\right)\right) .
$$

Then

$$
\begin{aligned}
\langle h(\underline{\boldsymbol{y}})\rangle_{\mathscr{C}} & \leq\left\langle\frac{1}{2} \log \left((2 \pi e)^{m} \operatorname{det}\left(I_{m}+\mathbb{E}\left[\boldsymbol{H Q}(\mathcal{C}) \boldsymbol{H}^{T}\right]\right)\right)\right\rangle_{\mathscr{C}} \\
& \stackrel{(a)}{\leq} \frac{1}{2} \log \left((2 \pi e)^{m} \operatorname{det}\left(I_{m}+\left\langle\mathbb{E}\left[\boldsymbol{H Q}(\mathcal{C}) \boldsymbol{H}^{T}\right]\right\rangle_{\mathscr{C}}\right)\right) \\
& \stackrel{(b)}{=} \frac{1}{2} \log \left((2 \pi e)^{m} \operatorname{det}\left(I_{m}+\mathbb{E}\left[\boldsymbol{H}\langle\mathrm{Q}(\mathcal{C})\rangle_{\mathscr{C}} \boldsymbol{H}^{T}\right]\right)\right) \\
& \stackrel{(c)}{=} \frac{1}{2} \log \left((2 \pi e)^{m} \operatorname{det}\left(I_{m}+P \mathbb{E}\left[\boldsymbol{H} \boldsymbol{H}^{T}\right]\right)\right),
\end{aligned}
$$

where $(a)$ is due to Jensen's inequality and concavity of $\log \operatorname{det}(\cdot),(b)$ is due to the Tonelli-Fubini Theorem enabling us to exchange the "averaging" operators $\mathbb{E}[\cdot]$ and $\langle\cdot\rangle_{\mathscr{C}}$ and $(c)$ is due to $\langle\mathrm{Q}(\mathcal{C})\rangle_{\mathscr{C}}=P I_{n}$. Note that

$$
\mathbb{E}\left[\left[\boldsymbol{H} \boldsymbol{H}^{T}\right]_{i, i}\right]=\sum_{\substack{l=1 \\ 0 \leq i-l \leq k}}^{n} \mathbb{E}\left[\boldsymbol{h}_{i, i-l}^{2}\right]=\sum_{\substack{l=1 \\ 0 \leq i-l \leq k}}^{n}\left(c_{i-l}^{2}+r_{i-l}^{2} / 3\right)
$$


and

$$
\mathbb{E}\left[\left[\boldsymbol{H} \boldsymbol{H}^{T}\right]_{i, j}\right]=\sum_{\substack{l=1 \\ 0 \leq i-l \leq k \\ 0 \leq j-l \leq n}}^{n} \mathbb{E}\left[\boldsymbol{h}_{i, i-l} \boldsymbol{h}_{j, j-l}\right]=\sum_{\substack{l=1 \\ 0 \leq i-l \leq k \\ 0 \leq j-l \leq k}}^{n} c_{i-l} c_{j-l}, \quad i \neq j .
$$

Let $H_{c}$ be the matrix given in (67). Then we can write

$$
\mathbb{E}\left[\boldsymbol{H} \boldsymbol{H}^{T}\right]=H_{c} H_{c}^{T}+D,
$$

where $D$ is a diagonal matrix with diagonal entries

$$
D_{i, i}=\sum_{\substack{l=1 \\ 0 \leq i-l \leq k}}^{n} r_{i-l}^{2} / 3 \leq\|\underline{r}\|_{2}^{2} / 3
$$

for every $1 \leq i \leq m$. By (115), (118) and (119),

$$
\begin{aligned}
\langle h(\underline{\boldsymbol{y}})\rangle_{\mathscr{C}} & \leq \frac{m}{2} \log (2 \pi e)+\frac{1}{2} \log \operatorname{det}\left(I_{m}+P H_{c} H_{c}^{T}+P\|\underline{r}\|_{2}^{2} / 3 I_{m}\right) \\
& \leq \frac{m}{2} \log (2 \pi e)+\frac{m}{2} \log \left(1+P\|\underline{r}\|_{2}^{2} / 3\right)+\frac{1}{2} \log \operatorname{det}\left(I_{m}+\frac{P}{1+P\|\underline{r}\|_{2}^{2} / 3} H_{c} H_{c}^{T}\right),
\end{aligned}
$$

where in the first step once again we have used the fact that $\log \operatorname{det}(\cdot)$ is nondecreasing along the cone of positive-semidefinite matrices.

B. Lower bound on $\langle h(\boldsymbol{y} \mid \underline{\boldsymbol{x}})\rangle_{\mathscr{C}}$

Fix a realization $\underline{x} \in \mathcal{C}$ for $\underline{x}$. Then

$$
\begin{aligned}
h(\underline{\boldsymbol{y}} \underline{\underline{\boldsymbol{x}}}=\underline{x}) & =h(\boldsymbol{H} \underline{\boldsymbol{x}}+\underline{\boldsymbol{z}} \mid \underline{\boldsymbol{x}}=\underline{x}) \\
& \stackrel{(a)}{=} h(\boldsymbol{H} \underline{x}+\underline{\boldsymbol{z}}) \\
& \stackrel{(b)}{=} h\left(\boldsymbol{H} \underline{x}-H_{c} \underline{x}+\underline{\boldsymbol{z}}\right) \\
& \stackrel{(c)}{=} h\left(\boldsymbol{E}_{c} \underline{x}+\underline{\boldsymbol{z}}\right) \\
& \stackrel{(d)}{=} \sum_{i=1}^{m} h\left(\left[\boldsymbol{E}_{c} \underline{x}\right]_{i}+\boldsymbol{z}_{i}\right) \\
& \stackrel{(e)}{\geq} \sum_{i=k+1}^{n} h\left(\left[\boldsymbol{E}_{c} \underline{x}\right]_{i}+\boldsymbol{z}_{i}\right),
\end{aligned}
$$

where $(a)$ is due to independence of $\underline{\boldsymbol{x}}$ and the pair $(\boldsymbol{H}, \underline{\boldsymbol{z}}),(b)$ is due to $H_{c} \underline{x}$ being a deterministic vector, the matrix $\boldsymbol{E}_{c}=\boldsymbol{H}-H_{c}$ in $(c)$ was originally defined in (78), (d) follows from independence of 
the entries of $\boldsymbol{E}_{c} \underline{x}+\underline{\boldsymbol{z}}$ and in step $(e)$ we have discarded the indices $1 \leq i \leq k$ and $n+1 \leq i \leq m$. For every $k+1 \leq i \leq n$, the random variable $\left[\boldsymbol{E}_{c} \underline{x}\right]_{i}=\sum_{j=i-k}^{i}\left[\boldsymbol{E}_{c}\right]_{i, j} x_{j}$ is the sum of (a constant number of) $k+1$ independent random variables. By entropy power inequality [20],

$$
\begin{aligned}
h\left(\left[\boldsymbol{E}_{c} \underline{x}\right]_{i}+\boldsymbol{z}_{i}\right) & \geq \frac{1}{2} \log \left(\sum_{j=i-k}^{i} 2^{2 h\left(\left[\boldsymbol{E}_{c}\right]_{i, j} x_{j}\right)}+2^{2 h\left(\boldsymbol{z}_{i}\right)}\right) \\
& \stackrel{(a)}{=} \frac{1}{2} \log \left(\sum_{j=i-k}^{i} 2^{2 \log \left(2 r_{i-j}\left|x_{j}\right|\right)}+2 \pi e\right) \\
& =\frac{1}{2} \log \left(\sum_{j=i-k}^{i} 4 r_{i-j}^{2} x_{j}^{2}+2 \pi e\right) \\
& =\frac{1}{2} \log \left(\sum_{j=0}^{k} 4 r_{j}^{2} x_{i-j}^{2}+2 \pi e\right) \\
& \stackrel{(b)}{\geq} \frac{1}{2} \max \left\{\log \sum_{j=0}^{k} 4 r_{j}^{2} x_{i-j}^{2}, \log (2 \pi e)\right\} \\
& \stackrel{(c)}{=} \frac{1}{2} \log (2 \pi e)+\frac{1}{2}\left(\log \sum_{j=0}^{k} \frac{2 r_{j}^{2} x_{i-j}^{2}}{\pi e}\right)
\end{aligned}
$$

where $(a)$ is due to $h\left(\boldsymbol{z}_{i}\right)=\frac{1}{2} \log (2 \pi e)$ and the fact that $\left[\boldsymbol{E}_{c}\right]_{i, j} x_{j}=\left(\boldsymbol{h}_{i, i-j}-c_{i-j}\right) x_{j}$ is uniformly distributed over the interval $\left[-r_{i-j}\left|x_{j}\right|, r_{i-j}\left|x_{j}\right|\right]$ for every $i, j$ where $0 \leq i-j \leq k,(b)$ is due to the trivial inequality $\log (a+b) \geq \max \{\log a, \log b\}$ and $(c)$ follows by adding and subtracting $\frac{1}{2} \log (2 \pi e)$. By (121) and (122) and averaging over $\underline{x} \in \mathscr{C}$,

$$
\begin{aligned}
\langle h(\underline{\boldsymbol{y}} \underline{\boldsymbol{x}})\rangle_{\mathscr{C}} & \geq \frac{1}{2}(n-k) \log (2 \pi e)+\frac{1}{2} \sum_{i=k+1}^{n}\left\langle\left(\log \sum_{j=0}^{k} \frac{2 r_{j}^{2} x_{i-j}^{2}}{\pi e}\right)^{+}\right\rangle_{\mathscr{C}} \\
& \geq \frac{1}{2}(n-k) \log (2 \pi e)+\frac{1}{2} \sum_{i=k+1}^{n}\left(\left\langle\log \sum_{j=0}^{k} \frac{2 r_{j}^{2} x_{i-j}^{2}}{\pi e}\right\rangle_{\mathscr{C}}\right)^{+},
\end{aligned}
$$

where the second step uses Jensen's inequality and convexity of the function $u^{+}=\max \{u, 0\}$ in terms of $u$. The term on the right side of (123) is an expectation involving a "weighted chi-square" random variable whose density does not admit a closed form. We further bound this term from below as follows. Let $\mathcal{J}=\left\{j: r_{j}>0\right\}$ and $k^{\prime}=|\mathcal{J}|$. Using arithmetic-geometric inequality,

$$
\begin{aligned}
\log \sum_{j=0}^{k} \frac{2 r_{j}^{2} x_{i-j}^{2}}{\pi e} & \geq \log \left(k^{\prime}\left(\prod_{j \in \mathcal{J}} \frac{2 r_{j}^{2} x_{i-j}^{2}}{\pi e}\right)^{\frac{1}{k^{\prime}}}\right) \\
& =\log k^{\prime}+\frac{1}{k^{\prime}} \sum_{j \in \mathcal{J}} \log \frac{2 r_{j}^{2} x_{i-j}^{2}}{\pi e}
\end{aligned}
$$


Then

$$
\begin{aligned}
\left\langle\log \sum_{j \in \mathcal{J}} \frac{2 r_{j}^{2} x_{i-j}^{2}}{\pi e}\right\rangle_{\mathscr{C}} & \geq \log k^{\prime}+\frac{1}{k^{\prime}} \sum_{j \in \mathcal{J}}\left\langle\log \frac{2 r_{j}^{2} x_{i-j}^{2}}{\pi e}\right\rangle_{\mathscr{C}} \\
& =\log k^{\prime}+\frac{1}{k^{\prime}} \sum_{j \in \mathcal{J}}\left(\log \frac{2 r_{j}^{2}}{\pi e}+\left\langle\log x_{i-j}^{2}\right\rangle_{\mathscr{C}}\right) .
\end{aligned}
$$

But,

$$
\begin{aligned}
\left\langle\log x_{i-j}^{2}\right\rangle_{\mathscr{C}} & =\int_{-\infty}^{\infty} p_{\mathrm{G}}(x ; P) \log x^{2} \mathrm{~d} x \\
& =\frac{2}{\sqrt{2 \pi P}} \int_{-\infty}^{\infty} e^{-\frac{x^{2}}{2 P}} \log |x| \mathrm{d} x \\
& =\log (2 P)+\frac{4}{\sqrt{\pi}} \int_{0}^{\infty} e^{-x^{2}} \log x \mathrm{~d} x
\end{aligned}
$$

where we have changed the variable $x$ to $\sqrt{2 P} x$. It is an easy exercise to show that

$$
\int_{0}^{\infty} e^{-x^{2}} \log x \mathrm{~d} x=-\frac{\sqrt{\pi}}{2}\left(\frac{1}{2 \ln 2} \gamma_{e}+1\right)
$$

where $\gamma_{e} \approx 0.577$ is the Euler-Mascheroni constant. Putting (125), (126) and (127) together,

$$
\left\langle\log \sum_{j \in \mathcal{J}} \frac{2 r_{j}^{2} x_{i-j}^{2}}{\pi e}\right\rangle_{\mathscr{C}} \geq \log \frac{P}{\pi e}-\frac{1}{\ln 2} \gamma_{e}+\log k^{\prime}+\frac{2}{k^{\prime}} \sum_{j \in \mathcal{J}} \log r_{j} .
$$

By (123) and (128) and denoting $\frac{1}{2} \log k^{\prime}+\frac{1}{k^{\prime}} \sum_{j \in \mathcal{J}}^{k} \log r_{j}$ by $\kappa^{\prime}$, we get

$$
\langle h(\underline{\boldsymbol{y}} \mid \underline{\boldsymbol{x}})\rangle_{\mathscr{C}} \geq \frac{1}{2}(n-k) \log (2 \pi e)+(n-k)\left(\frac{1}{2} \log \frac{P}{\pi e}+\kappa^{\prime}-\frac{1}{2 \ln 2} \gamma_{e}\right)^{+} .
$$

Putting (120) and (129) together,

$$
\begin{gathered}
\langle I(\underline{\boldsymbol{x}} ; \underline{\boldsymbol{y}})\rangle_{\mathscr{C}} \leq k \log (2 \pi e)+\frac{m}{2} \log \left(1+P\|\underline{r}\|_{2}^{2} / 3\right)+\frac{1}{2} \log \operatorname{det}\left(I_{m}+\frac{P}{1+\|\underline{r}\|_{2}^{2} P / 3} H_{c} H_{c}^{T}\right) \\
-(n-k)\left(\frac{1}{2} \log \frac{P}{\pi e}+\kappa^{\prime}-\frac{1}{2 \ln 2} \gamma_{e}\right)^{+} .
\end{gathered}
$$

The desired upper bound in (31) follows from (109) and (130) and the facts that $\lim _{n \rightarrow \infty}\left\langle p_{e}(\mathcal{C})\right\rangle_{\mathscr{C}}=0$ and $\lim _{n \rightarrow \infty} \frac{1}{2 m} \log \operatorname{det}\left(I_{m}+\frac{P}{1+P\|\underline{\|}\|_{2}^{2} / 3} H_{c} H_{c}^{T}\right)=C_{W G I, 0}\left(\frac{P}{1+P\|\underline{\|}\|_{2}^{2} / 3}\right)$.

We end this appendix by two remarks:

(i) The constant $\kappa^{\prime}$ in (130) can be replaced by $\kappa=\max \left\{\kappa^{\prime}, \log \|\underline{r}\|_{\infty}\right\}$ defined in (30). This follows 
from a different lower bound on the term on the right side of (124) given by

$$
\log \sum_{j=0}^{k} \frac{2 r_{j}^{2} x_{i-j}^{2}}{\pi e} \geq \log \frac{2\|\underline{r}\|_{\infty}^{2} x_{i-j_{0}}^{2}}{\pi e}=\log \frac{2\|\underline{r}\|_{\infty}^{2}}{\pi e}+\log x_{i-j_{0}}^{2}
$$

where $0 \leq j_{0} \leq k$ is such that $r_{j_{0}}=\|\underline{r}\|_{\infty}$. The term $\left\langle\log x_{i-j_{0}}^{2}\right\rangle_{\mathscr{C}}$ can be computed as in (126).

(ii) Assume there is $r>0$ such that $r_{i}=r$ for every $0 \leq i \leq k$. Then

$$
\left\langle\log \sum_{j=0}^{k} \frac{2 r_{j}^{2} x_{i-j}^{2}}{\pi e}\right\rangle_{\mathscr{C}}=\log \frac{2 r^{2}}{\pi e}+\left\langle\log \sum_{j=0}^{k} x_{i-j}^{2}\right\rangle_{\mathscr{C}}=\log \frac{2 r^{2}}{\pi e}+\log P+\mathbb{E}\left[\log \chi_{k+1}^{2}\right]
$$

\section{APPENDix C; PROOF OF (63)}

For a random variable $\boldsymbol{x}$ and a measurable function $f$, we adopt the notation

$$
\mathbb{E}_{\boldsymbol{x}}[f(x)]=\mathbb{E}[f(\boldsymbol{x})]
$$

For notational simplicity, we denote $\boldsymbol{\Phi}_{\tau}$ by $\boldsymbol{\Phi}$ through this appendix. By definition,

$$
\operatorname{Var}\left(\underline{\boldsymbol{\nu}}^{T} \boldsymbol{\Phi} \underline{\boldsymbol{\nu}}\right)=\mathbb{E}\left[\left(\underline{\boldsymbol{\nu}}^{T} \boldsymbol{\Phi} \underline{\boldsymbol{\nu}}\right)^{2}\right]-\left(\mathbb{E}\left[\underline{\boldsymbol{\nu}}^{T} \boldsymbol{\Phi} \underline{\boldsymbol{\nu}}\right]\right)^{2}
$$

But,

$$
\begin{aligned}
& \mathbb{E}\left[\left(\underline{\boldsymbol{\nu}}^{T} \boldsymbol{\Phi} \underline{\boldsymbol{\nu}}\right)^{2}\right] \stackrel{(a)}{=} \mathbb{E}_{\boldsymbol{\Phi}}\left[\mathbb{E}\left[\left(\underline{\boldsymbol{\nu}}^{T} \boldsymbol{\Phi} \underline{\boldsymbol{\nu}}\right)^{2} \mid \boldsymbol{\Phi}=\Phi\right]\right] \\
& \stackrel{(b)}{=} \mathbb{E}_{\boldsymbol{\Phi}}\left[\mathbb{E}\left[\left(\underline{\boldsymbol{\nu}}^{T} \Phi \underline{\boldsymbol{\nu}}\right)^{2}\right]\right] \\
& \stackrel{(c)}{=} \quad \mathbb{E}_{\boldsymbol{\Phi}}\left[\operatorname{Var}\left(\underline{\boldsymbol{\nu}}^{T} \Phi \underline{\boldsymbol{\nu}}\right)+\left(\mathbb{E}\left[\underline{\boldsymbol{\nu}}^{T} \Phi \underline{\boldsymbol{\nu}}\right]\right)^{2}\right] \\
& \stackrel{(d)}{=} \quad \mathbb{E}_{\boldsymbol{\Phi}}\left[2 \operatorname{tr}\left(\Phi^{2}\right)+(\operatorname{tr}(\Phi))^{2}\right] \\
& \stackrel{(e)}{=} 2 \mathbb{E}\left[\operatorname{tr}\left(\boldsymbol{\Phi}^{2}\right)\right]+\mathbb{E}\left[(\operatorname{tr}(\boldsymbol{\Phi}))^{2}\right],
\end{aligned}
$$

where $(a)$ is due to the tower property of expectation, i.e., $\mathbb{E}[\boldsymbol{x}]=\mathbb{E}_{\boldsymbol{y}}[\mathbb{E}[\boldsymbol{x} \mid \boldsymbol{y}=y]]$, (b) is due to independence of $\boldsymbol{\nu}$ and $\boldsymbol{\Phi},(c)$ is due to the definition of variance, $(d)$ is due to the well-known formula $\operatorname{Var}\left(\underline{\boldsymbol{\nu}}^{T} Q \underline{\boldsymbol{\nu}}\right)=2 \operatorname{tr}\left(Q^{2}\right)$ for arbitrary square matrix $Q$ and $(e)$ is due to the notation adopted in (133). Moreover, we have seen earlier in (60) that $\mathbb{E}\left[\underline{\boldsymbol{\nu}}^{T} \boldsymbol{\Phi} \underline{\boldsymbol{\nu}}\right]=\mathbb{E}[\operatorname{tr}(\boldsymbol{\Phi})]$. Using this together with (135), the proof of (63) is complete. 


\section{APPENDIX D; PROOF OF (64)}

We need the following lemma:

Lemma 5. For a matrix $M$, define the maximum row-sum norm by

$$
\|M\|_{r}:=\max _{i} \sum_{j}\left|M_{i, j}\right|
$$

Then $\|\cdot\|_{r}$ becomes a matrix norm. In particular, it is sub-multiplicative, i.e.,

$$
\left\|M_{1} M_{2}\right\|_{r} \leq\left\|M_{1}\right\|_{r}\left\|M_{2}\right\|_{r}
$$

for every two matrices $M_{1}$ and $M_{2}$ of proper sizes. Moreover,

$$
\|M\|^{2} \leq\left\|M^{T}\right\|_{r}\|M\|_{r}
$$

for every matrix $M$.

Proof. See [18]. The statement in (138) is a special case of the fact that the spectral radius of a square matrix is bounded from above by every matrix-norm of that matrix.

Fix a realization $\Phi_{\tau}$ of $\boldsymbol{\Phi}_{\tau}$. We proceed to find an upper bound on $\operatorname{tr}\left(\Phi_{\tau}^{2}\right)$. We write

$$
\operatorname{tr}\left(\Phi_{\tau}^{2}\right) \leq(m+n) \lambda_{\max }\left(\Phi_{\tau}^{2}\right)=(m+n)\|\Phi\|^{2} \leq(m+n)\left\|\Phi_{\tau}\right\|_{r}^{2}
$$

where the last step is due to (138) in Lemma 5. Looking at the structure of $\Phi$ in (53),

$$
\begin{aligned}
& \|\Phi\|_{r}=\max \left\{\left\|\left[\begin{array}{ll}
P E_{\tau}^{T} E_{\tau} & \sqrt{P} E_{\tau}^{T}
\end{array}\right]\right\|_{r},\left\|\left[\begin{array}{cc}
\sqrt{P} E_{\tau} & I_{n}
\end{array}\right]\right\|_{r}\right\} \\
& \leq \max \left\{P\left\|E_{\tau}^{T} E_{\tau}\right\|_{r}+\sqrt{P}\left\|E_{\tau}^{T}\right\|_{r}, 1+\sqrt{P}\left\|E_{\tau}\right\|_{r}\right\} \\
& \leq \max \left\{P\left\|E_{\tau}^{T}\right\|_{r}\left\|E_{\tau}\right\|_{r}+\sqrt{P}\left\|E_{\tau}^{T}\right\|_{r}, 1+\sqrt{P}\left\|E_{\tau}\right\|_{r}\right\},
\end{aligned}
$$

where the penultimate step is due to the fact that $\|M\|_{r} \leq\left\|M_{1}\right\|_{r}+\left\|M_{2}\right\|_{r}$ for a block matrix $M=$ $\left[\begin{array}{ll}M_{1} & M_{2}\end{array}\right]$ and the last step is due to (137) in Lemma 5. The matrix $E_{\tau}$ is banded, i.e., $\left[E_{\tau}\right]_{i, j}=0$ for $i-j<0$ or $i-j>k$ and $\left|\left[E_{\tau}\right]_{i, j}\right| \leq \frac{1}{2} \tau_{i-j}$ for $0 \leq i-j \leq k$. It follows that

$$
\left\|E_{\tau}\right\|_{r} \leq \frac{1}{2} \sum_{l=0}^{k} \tau_{l}, \quad\left\|E_{\tau}^{T}\right\|_{r} \leq \frac{1}{2} \sum_{l=0}^{k} \tau_{l} .
$$


It is clear that $\frac{1}{2} \sum_{l=0}^{k} \tau_{l} \leq \sum_{l=0}^{k} r_{l}=r_{s}$. Putting (140) and (141) together,

$$
\left\|\Phi_{\tau}\right\|_{r} \leq \max \left\{r_{s}^{2} P+r_{s} \sqrt{P}, 1+r_{s} \sqrt{P}\right\} \leq r_{s}^{2} P+r_{s} \sqrt{P}+1
$$

By (139) and (142),

$$
\operatorname{tr}\left(\Phi_{\tau}^{2}\right) \leq\left(r_{s}^{2} P+r_{s} \sqrt{P}+1\right)^{2}(m+n)
$$

Appendix E; ProOf OF LEMMA 2

We write

$$
\begin{aligned}
\left\|\Omega_{c}^{-1} H_{c}\right\|_{2}^{2} & =\operatorname{tr}\left(\left(\Omega_{c}^{-1} H_{c}\right)^{T} \Omega_{c}^{-1} H_{c}\right) \\
& \leq n \lambda_{\max }\left(\left(\Omega_{c}^{-1} H_{c}\right)^{T} \Omega_{c}^{-1} H_{c}\right) \\
& =n \lambda_{\max }\left(H_{c}^{T} \Omega_{c}^{-2} H_{c}\right) .
\end{aligned}
$$

Let

$$
H_{c}=U \Lambda V^{T}
$$

be the singular value decomposition for $H_{c}$. Here, $U$ and $V$ are $m \times m$ and $n \times n$ real orthogonal matrices and

$$
\Lambda=\left[\begin{array}{c}
\Lambda_{c} \\
0_{k, n}
\end{array}\right],
$$

where $\Lambda_{c}$ is an $n \times n$ diagonal matrix whose diagonal entries are the nonnegative square roots of the eigenvalues of $H_{c}^{T} H_{c}$. Then

$$
H_{c}^{T} \Omega_{c}^{-2} H_{c}=V \Lambda^{T}\left(I_{m}+P \Lambda \Lambda^{T}\right)^{-2} \Lambda V^{T}
$$

and we get

$$
\lambda_{\max }\left(H_{c}^{T} \Omega_{c}^{-2} H_{c}\right)=\lambda_{\max }\left(\Lambda^{T}\left(I_{m}+P \Lambda \Lambda^{T}\right)^{-2} \Lambda\right) .
$$

By (146),

$$
\Lambda^{T}\left(I_{m}+P \Lambda \Lambda^{T}\right)^{-2} \Lambda=\Lambda_{c}^{2}\left(I_{n}+P \Lambda_{c}^{2}\right)^{-2}
$$


As such,

$$
\lambda_{\max }\left(H_{c}^{T} \Omega_{c}^{-2} H_{c}\right)=\max \left\{\frac{\lambda}{(1+P \lambda)^{2}}: \lambda \text { is an eigenvalue of } H_{c}^{T} H_{c}\right\} .
$$

We need the following lemma which is a restatement of Lemma 4.1 in [19]:

Lemma 6. Let $T$ be an $n \times n$ symmetric Toeplitz matrix with real entries given by $T_{i, j}=t_{i-j}$ where $t_{-l}=t_{l}$ for every $0 \leq l \leq n-1$. Moreover, define the function $f_{T}(\cdot)$ by

$$
f_{T}(\omega)=\sum_{l=-(n-1)}^{n-1} t_{l} e^{\sqrt{-1} l \omega}, \quad 0 \leq \omega \leq 2 \pi
$$

Then every eigenvalue of $T$ lies in the interval $\left[\min _{\omega} f_{T}(\omega), \max _{\omega} f_{T}(\omega)\right]$.

The matrix $H_{c}^{T} H_{c}$ is a symmetric Toeplitz matrix given by $\left[H_{c}^{T} H_{c}\right]_{i, j}=\sum_{l=0}^{k-|i-j|} c_{l} c_{l+|i-j|} \mathbb{1}_{|i-j| \leq k}$. Then $f_{H_{c}^{T} H_{c}}(\omega)=\left|\sum_{i=0}^{k} c_{i} e^{\sqrt{-1} i \omega}\right|^{2}$. By Lemma 6, every eigenvalue $\lambda$ of $H_{c}^{T} H_{c}$ satisfies

$$
\alpha^{2} \leq \lambda \leq \beta^{2}
$$

where $\alpha$ and $\beta$ are given in (19). The function $\frac{x}{(1+P x)^{2}}$ is increasing for $0<x \leq \frac{1}{P}$ and decreasing for $x \geq \frac{1}{P}$. Using this fact together with (150) and (152),

$$
\lambda_{\max }\left(H_{c}^{T} \Omega_{c}^{-2} H_{c}\right) \leq \begin{cases}\frac{\beta^{2}}{\left(1+P \beta^{2}\right)^{2}} & \frac{1}{P} \geq \beta^{2} \\ \frac{\alpha^{2}}{\left(1+P \alpha^{2}\right)^{2}} & \frac{1}{P} \leq \alpha^{2}\end{cases}
$$

For values of $P$ satisfying $\alpha^{2}<\frac{1}{P}<\beta^{2}$, we simply let

$$
\lambda_{\max }\left(H_{c}^{T} \Omega_{c}^{-2} H_{c}\right) \leq \sup _{\lambda \geq 0} \frac{\lambda}{(1+P \lambda)^{2}}=\frac{1}{4 P}
$$

By (144), (153) and (154) and recalling the definition of $\varrho$ in (20), we get $\left\|\Omega_{c}^{-1} H_{c}\right\|_{2} \leq \sqrt{n} \varrho$. 


\section{APPENDIX F; PROOF OF (87)}

We compute an upper bound on $\operatorname{tr}\left(\Psi^{2}\right)$ where the positive semidefinite matrix $\Psi$ is a fixed realization of $\Psi$ in (75). It appears useless to start as in (139) due to the fact that we do not have an upper bound on $\left\|\Omega_{c}^{-1}\right\|_{r}$. The following lemma proves helpful:

Lemma 7. Let the partitioned symmetric matrix $M$ be given by

$$
M=\left[\begin{array}{cc}
A & B^{T} \\
B & C
\end{array}\right]
$$

Then

$$
\|M\| \leq \max \{\|A\|+\|B\|,\|C\|+\|B\|\} .
$$

Proof. Let us write the matrix $M$ in (155) as

$$
M=M_{1}+M_{2},
$$

with

$$
M_{1}=\left[\begin{array}{cc}
A & O_{1}^{T} \\
O_{1} & C
\end{array}\right], \quad M_{2}=\left[\begin{array}{cc}
O_{2} & B^{T} \\
B & O_{3}
\end{array}\right],
$$

where $O_{1}, O_{2}$ and $O_{3}$ are zero matrices of appropriate sizes. It is well-known that

$$
\left\|M_{1}\right\|=\max \{\|A\|,\|C\|\}, \quad\left\|M_{2}\right\|=\|B\| .
$$

Hence,

$$
\|M\| \leq\left\|M_{1}\right\|+\left\|M_{2}\right\|=\max \{\|A\|+\|B\|,\|C\|+\|B\|\} .
$$

Let us write

$$
\Psi=\left[\begin{array}{ll}
\Psi_{1,1} & \Psi_{1,2} \\
\Psi_{1,2}^{T} & \Psi_{2,2}
\end{array}\right]
$$


where

$$
\Psi_{1,1}:=P H^{T} \Omega_{c}^{-1} H \quad \Psi_{1,2}:=\sqrt{P} H^{T} \Omega_{c}^{-1}, \quad \Psi_{2,2}:=\Omega_{c}^{-1}
$$

Then

$\operatorname{tr}\left(\Psi^{2}\right) \leq(m+n) \lambda_{\max }\left(\Psi^{2}\right)=(m+n)\|\Psi\|^{2} \leq(m+n)\left(\max \left\{\left\|\Psi_{1,1}\right\|+\left\|\Psi_{1,2}\right\|,\left\|\Psi_{2,2}\right\|+\left\|\Psi_{1,2}\right\|\right\}\right)^{2}$

where last step is due to Lemma 7. But,

$$
\left\|\Psi_{1,1}\right\|=\left\|P H^{T} \Omega_{c}^{-1} H\right\| \leq P\left\|H^{T}\right\|\left\|\Omega_{c}^{-1}\right\|\|H\| \leq P\|H\|^{2},
$$

where we have used the facts that $\left\|H^{T}\right\|=\|H\|$ and $\left\|\Omega_{c}^{-1}\right\| \leq 1$. Similarly,

$$
\left\|\Psi_{1,2}\right\| \leq \sqrt{P}\|H\|
$$

and

$$
\left\|\Psi_{2,2}\right\| \leq 1
$$

By (163), (164), (165) and (166),

$$
\begin{aligned}
\operatorname{tr}\left(\Psi^{2}\right) & \leq(m+n)\left(\max \left\{P\|H\|^{2}+\sqrt{P}\|H\|, 1+\sqrt{P}\|H\|\right\}\right)^{2} \\
& \leq(m+n)\left(P\|H\|^{2}+\sqrt{P}\|H\|+1\right)^{2}
\end{aligned}
$$

The matrix $H$ is banded, i.e., $H_{i, j}=0$ for $i-j<0$ or $i-j>k$. Moreover, $\left|H_{i, j}\right|=\left|h_{i, i-j}\right| \leq\left|c_{i-j}\right|+r_{i-j}$ for $0 \leq i-j \leq k$. It follows that

$$
\|H\|_{r} \leq c_{s}+r_{s}, \quad\left\|H^{T}\right\|_{r} \leq c_{s}+r_{s}
$$

Using (168) together with (138) in Lemma 5, we get

$$
\|H\| \leq\left(\|H\|_{r}\left\|H^{T}\right\|_{r}\right)^{1 / 2} \leq c_{s}+r_{s} .
$$


By (167) and (169),

$$
\operatorname{tr}\left(\Psi^{2}\right) \leq(m+n)\left(\left(c_{s}+r_{s}\right)^{2} P+\left(c_{s}+r_{s}\right) \sqrt{P}+1\right)^{2}
$$

\section{APPENDiX G; PROOF OF LEMMA 3}

Fix $H \in \mathcal{H}$. In this appendix we obtain three lower bounds on $\frac{\operatorname{det}\left(\Omega_{H}\right)}{\operatorname{det}\left(\Omega_{c}\right)}$. In general, none of these bounds is tighter than the other two. We prove (95) first. Recall the matrix $E_{c}$ in (78). Writing $H=E_{c}+H_{c}$,

$$
\begin{aligned}
\operatorname{det}\left(\Omega_{H}\right) & =\operatorname{det}\left(I_{m}+P H H^{T}\right) \\
& =\operatorname{det}\left(I_{m}+P H_{c} H_{c}^{T}+P E_{c} E_{c}^{T}+P E_{c} H_{c}^{T}+P H_{c} E_{c}^{T}\right) \\
& =\operatorname{det}\left(\Omega_{c}\right) \operatorname{det}\left(I_{m}+\Delta\right),
\end{aligned}
$$

where

$$
\Delta:=\Omega_{c}^{-1} \Omega_{H}-I_{m}=P \Omega_{c}^{-1} E_{c} E_{c}^{T}+P \Omega_{c}^{-1} E_{c} H_{c}^{T}+P \Omega_{c}^{-1} H_{c} E_{c}^{T}
$$

We make two observations about the matrix $\Delta$ :

(i) The matrix $\Omega_{c}^{-1} \Omega_{H}$ is a product of two positive-definite matrices and hence, its eigenvalues are all real (and positive). Therefore, eigenvalues of $\Delta$ are all real numbers as well.

(ii) The spectral norm $\|\Delta\|$ is bounded as

$$
\begin{aligned}
\|\Delta\| & \leq P\left\|\Omega_{c}^{-1}\right\|\left\|E_{c}\right\|\left\|E_{c}^{T}\right\|+P\left\|\Omega_{c}^{-1}\right\|\left\|E_{c}\right\|\left\|H_{c}^{T}\right\|+P\left\|\Omega_{c}^{-1} H_{c}\right\|\left\|E_{c}^{T}\right\| \\
& \stackrel{(a)}{\leq} P\left\|E_{c}\right\|^{2}+P\left\|E_{c}\right\|\left\|H_{c}\right\|+P\left\|\Omega_{c}^{-1} H_{c}\right\|\left\|E_{c}\right\| \\
& \stackrel{(b)}{\leq} r_{s}^{2}+P r_{s}\left\|H_{c}\right\|+P r_{s}\left\|\Omega_{c}^{-1} H_{c}\right\| \\
& \stackrel{(c)}{\leq} \operatorname{Pr}_{s}^{2}+\operatorname{Pr}_{s} \beta+\operatorname{Pr}_{s}\left\|\Omega_{c}^{-1} H_{c}\right\| \\
& \stackrel{(d)}{\leq} \operatorname{Pr}_{s}^{2}+\operatorname{Pr}_{s} \beta+P r_{s} \varrho \\
& =r_{s}\left(r_{s}+\beta+\varrho\right) P,
\end{aligned}
$$

where $(a)$ is due to $\left\|M^{T}\right\|=\|M\|$ for every matrix $M$ and $\left\|\Omega_{c}^{-1}\right\| \leq 1,(b)$ is due to $\left\|E_{c}\right\| \leq r_{s}$ which itself is a consequence of $\left\|E_{c}\right\|_{r} \leq r_{s},\left\|E_{c}^{T}\right\| \leq r_{s}$ and (138) in Lemma 5, (c) is due to $\left\|H_{c}\right\| \leq \beta$ which follows from (152) and $(d)$ is due to (153), (154) and the definition of $\varrho$ in (20). 
It results from (i) and (ii) that no eigenvalue of $\Delta$ is larger than $r_{s}\left(r_{s}+\beta+\varrho\right) P$ in absolute value. Therefore, $\operatorname{det}\left(I_{m}+\Delta\right) \geq\left(1-r_{s}\left(r_{s}+\beta+\varrho\right) P\right)^{m}$ where we have assumed $r_{s}$ is small enough such that $r_{s}\left(r_{s}+\beta+\varrho\right) P<1$. Using this in (171),

$$
\operatorname{det}\left(\Omega_{H}\right) \geq \operatorname{det}\left(\Omega_{c}\right)\left(1-r_{s}\left(r_{s}+\beta+\varrho\right) P\right)^{m}
$$

Next, we develop a second lower bound on $\operatorname{det}\left(\Omega_{H}\right)$. For a real symmetric $n \times n$ matrix $X$, denote its eigenvalues in increasing order by $\lambda_{i}(X)$ for $1 \leq i \leq n$, i.e., $\lambda_{1}(X) \leq \lambda_{2}(X) \leq \cdots \leq \lambda_{n}(X)$. We need the following lemma which is a direct consequence of Theorem 4.3.1 in [18] due to Weyl:

Lemma 8. Let $A$ and $B$ be $n \times n$ real symmetric matrices. Then

$$
\left|\lambda_{i}(A)-\lambda_{i}(B)\right| \leq\|A-B\|
$$

for every $1 \leq i \leq n$.

Denote the eigenvalues of $H^{T} H$ and $H_{c}^{T} H_{c}$ in increasing order by $\mu_{i}$ and $\lambda_{i}$, respectively. Then

$$
\frac{\operatorname{det}\left(\Omega_{H}\right)}{\operatorname{det}\left(\Omega_{c}\right)}=\frac{\operatorname{det}\left(I_{m}+P H H^{T}\right)}{\operatorname{det}\left(I_{m}+P H_{c} H_{c}^{T}\right)}=\frac{\operatorname{det}\left(I_{n}+P H^{T} H\right)}{\operatorname{det}\left(I_{n}+P H_{c}^{T} H_{c}\right)}=\prod_{i=1}^{n} \frac{1+P \mu_{i}}{1+P \lambda_{i}} .
$$

We can write

$$
\frac{1+P \mu_{i}}{1+P \lambda_{i}}=1+\frac{P\left(\mu_{i}-\lambda_{i}\right)}{1+P \lambda_{i}} \geq 1-\frac{P\left|\mu_{i}-\lambda_{i}\right|}{1+P \min _{i} \lambda_{i}},
$$

for every $1 \leq i \leq n$. By (152), $\min _{i} \lambda_{i} \geq \alpha^{2}$ where $\alpha$ is given in (19). Moreover, $\left|\mu_{i}-\lambda_{i}\right| \leq \| H^{T} H-$ $H_{c}^{T} H_{c} \|$ due to Lemma 8 . Therefore,

$$
\frac{1+P \mu_{i}}{1+P \lambda_{i}} \geq 1-\frac{P\left\|H^{T} H-H_{c}^{T} H_{c}\right\|}{1+\alpha^{2} P}
$$

Writing $H=E_{c}+H_{c}$, we get

$$
\left\|H^{T} H-H_{c}^{T} H_{c}\right\|=\left\|E_{c}^{T} E_{c}+E_{c}^{T} H_{c}+H_{c}^{T} E_{c}\right\| \leq\left\|E_{c}\right\|^{2}+2\left\|E_{c}\right\|\left\|H_{c}\right\| \leq r_{s}^{2}+2 r_{s} \beta,
$$

where the last step is due to $\left\|H_{c}\right\| \leq \beta$ and $\|E\| \leq r_{s}$. By (176), (177), (178) and (179)

$$
\operatorname{det}\left(\Omega_{H}\right) \geq \operatorname{det}\left(\Omega_{c}\right)\left(1-\frac{r_{s}\left(r_{s}+2 \beta\right) P}{1+\alpha^{2} P}\right)^{n}
$$


where we have assumed $r_{s}$ is small enough such that $\frac{r_{s}\left(r_{s}+2 \beta\right) P}{1+\alpha^{2} P}<1$.

Finally, we prove (94). Define

$$
\Omega(t)=I_{m}+P\left(H_{c}+t E_{c}\right)\left(H_{c}+t E_{c}\right)^{T}
$$

and

$$
F(t)=\ln \operatorname{det}(\Omega(t)),
$$

where $0 \leq t \leq 1$. The function $F$ is differentiable on $[0,1]$ and we can apply the Mean Value Theorem to write

$$
F(1)-F(0)=F^{\prime}(u)
$$

for some $u \in(0,1)$. Note that $\Omega(0)=\Omega_{c}$ and $\Omega(1)=\Omega_{H}$. Therefore, one has the bound

$$
\left|\ln \operatorname{det}\left(\Omega_{H}\right)-\ln \operatorname{det}\left(\Omega_{c}\right)\right| \leq \max _{0 \leq t \leq 1}\left|F^{\prime}(t)\right|
$$

Using the formula [16]

$$
\mathrm{d}(\ln \operatorname{det}(A))=\operatorname{tr}\left(A^{-1} \mathrm{~d} A\right)
$$

for any differentiable positive-definite matrix $A$,

$$
F^{\prime}(t)=\operatorname{tr}\left(\Omega^{-1}(t) \Omega^{\prime}(t)\right)
$$

where

$$
\Omega^{\prime}(t)=\frac{d}{d t} \Omega(t)=P H_{c} E_{c}^{T}+P E_{c} H_{c}^{T}+2 t P E_{c} E_{c}^{T} .
$$

By Cauchy-Schwarz inequality,

$$
\left|F^{\prime}(t)\right|=\left|\operatorname{tr}\left(\Omega^{-1}(t) \Omega^{\prime}(t)\right)\right| \leq\left\|\Omega^{-1}(t)\right\|_{2}\left\|\Omega^{\prime}(t)\right\|_{2} .
$$

We have

$$
\left\|\Omega^{-1}(t)\right\|_{2}^{2}=\operatorname{tr}\left(\Omega^{-2}(t)\right) \leq m \lambda_{\max }\left(\Omega^{-2}(t)\right) \leq m
$$


where the last step is due to the fact that no eigenvalue of $\Omega^{-1}(t)$ is larger than one. Also,

$$
\begin{aligned}
\left\|\Omega^{\prime}(t)\right\|_{2}^{2} & =\operatorname{tr}\left(\left(\Omega^{\prime}(t)\right)^{2}\right) \\
& \leq m \lambda_{\max }\left(\left(\Omega^{\prime}(t)\right)^{2}\right) \\
& =m \lambda_{\max }^{2}\left(\Omega^{\prime}(t)\right) \\
& \leq m\left\|\Omega^{\prime}(t)\right\|^{2} \\
& \leq m\left(2 r_{s}\left(r_{s}+\beta\right) P\right)^{2},
\end{aligned}
$$

where once again we have used $\left\|H_{c}\right\| \leq \beta$ and $\left\|E_{c}\right\| \leq r_{s}$. By (184), (188), (189) and (190),

$$
\left|\log \operatorname{det}\left(\Omega_{H}\right)-\log \operatorname{det}\left(\Omega_{c}\right)\right| \leq \frac{2 m}{\ln 2} r_{s}\left(r_{s}+\beta\right) P
$$

\section{APPENDiX H; PROOF OF LEMMA 4}

We write

$$
\inf _{\underline{y} \in \mathcal{T}_{\eta^{\prime}}^{(m)}\left(\Omega_{c}\right)} \underline{y}^{T} \Omega_{H}^{-1} \underline{y}=\inf _{m\left(1-\eta^{\prime}\right)<t<m\left(1+\eta^{\prime}\right)} \min _{\underline{y} \in \mathbb{R}^{m}: \underline{y}^{T} \Omega_{c}^{-1} \underline{y}=t} \underline{y}^{T} \Omega_{H}^{-1} \underline{y} .
$$

To achieve min $y \in \mathbb{R}^{m} \quad \underline{y}^{T} \Omega_{H}^{-1} \underline{y}$, the gradient of $\underline{y}^{T} \Omega_{H}^{-1} \underline{y}$ must be parallel to the gradient of $\underline{y}^{T} \Omega_{c}^{-1} \underline{y}$, i.e., $\underline{y}^{T} \Omega_{c}^{-1} \underline{y}=t$

$$
\Omega_{H}^{-1} \underline{y}=\lambda \Omega_{c}^{-1} \underline{y}
$$

where $\lambda$ is the Lagrange multiplier. This says that the "optimum" $y$ must be an eigenvector for the matrix $\Omega_{c} \Omega_{H}^{-1}$ with corresponding eigenvalue $\lambda$. Multiplying both sides of (193) by $\underline{y}^{T}$ from left, we conclude that

$$
\inf _{\underline{y} \in \mathbb{R}^{m}: \underline{y}^{T} \Omega_{c}^{-1} \underline{y}_{y}=t} \underline{y}^{T} \Omega_{H}^{-1} \underline{y}=t \lambda_{\min }\left(\Omega_{c} \Omega_{H}^{-1}\right),
$$

where $\lambda_{\min }\left(\Omega_{c} \Omega_{H}^{-1}\right)$ is the minimum eigenvalue of $\Omega_{c} \Omega_{H}^{-1}$. Putting (192) and (194) together,

$$
\inf _{\underline{y} \in \mathcal{T}_{\eta^{\prime}}^{(m)}\left(\Omega_{c}\right)} \underline{y}^{T} \Omega_{H}^{-1} \underline{y}=m\left(1-\eta^{\prime}\right) \lambda_{\min }\left(\Omega_{c} \Omega_{H}^{-1}\right) .
$$




\section{Moreover,}

$$
\begin{aligned}
\lambda_{\min }\left(\Omega_{c} \Omega_{H}^{-1}\right) & =\frac{1}{\lambda_{\max }\left(\Omega_{c}^{-1} \Omega_{H}\right)} \\
& \stackrel{(a)}{=} \frac{1}{\lambda_{\max }\left(I_{m}+\Delta\right)} \\
& \stackrel{(b)}{\geq} \frac{1}{1+\|\Delta\|} \\
& \stackrel{(c)}{\geq} \phi_{5},
\end{aligned}
$$

where $(a)$ is due to the definition of $\Delta$ in (172), (b) is due to $\|\Delta\|$ being an upper bound on $\lambda_{\max }(\Delta)$ and (c) is due to (173) and the definition of $\phi_{5}$.

\section{REFERENCES}

[1] J. G. Proakis and M. Salehi, "Digital communications (5th edition)", McGraw-Hill, 2007.

[2] W. Hirt and J. L. Massey, "Capacity of the discrete-time Gaussian channel with intersymbol interference", IEEE Trans. Inf. Theory, vol. 34, no. 3, pp. 380-388, May 1988.

[3] S. Shamai (Shitz) and R. Laroia, "The intersymbol interference channel: Lower bounds on capacity and channel precoding loss", IEEE Trans. Inf. Theory, vol. 42, no. 5, pp. 1388-1404, Sept. 1996.

[4] Y. Carmon and S. Shamai (Shitz), "Lower bounds and approximations for the information rate of the ISI channel", IEEE Trans. Inf. Theory, vol. 61, no. 10, pp. 5417-5431, Oct. 2015.

[5] Y. Carmon, S. Shamai (Shitz) and T. Weissman, "Comparison of the achievable rates in OFDM and single carrier modulation with I.I.D. Inputs", IEEE Trans. Inf. Theory, vol. 61, no. 4, pp. 1795-1818, April 2015.

[6] N. Merhav, "Universal decoding for memoryless Gaussian channels with a deterministic interference", IEEE Trans. Inf. Theory, vol. 39, no. 4, pp. 1261-1269, July 1993.

[7] J. Ziv, "Universal decoding for finite-state channels", IEEE Trans. Inf. Theory, vol. 31, no. 4, pp. 453-460, July 1985.

[8] M. Feder and A. Lapidoth, "Universal decoding for channels with memory", IEEE Trans. Inf. Theory, vol. 44, no. 5, pp. 1726-1745, Sept. 1998.

[9] M. Feder and N. Merhav, "Universal composite hypothesis testing: A competitive minimax approach", IEEE Trans. Inf. Theory, vol. 48, no. 6, pp. 1504-1517, June 2002.

[10] W. Huleihel and N. Merhav, "Universal decoding for Gaussian intersymbol interference channels", IEEE Trans. Inf. Theory, vol. 61, no. 4, pp. 1606-1618, April 2015.

[11] S. N. Diggavi, "On achievable performance of spatial diversity fading channels", IEEE Trans. Inf. Theory, vol. 47, no. 1, pp. 308-325, January 2001.

[12] A. J. Goldsmith and M. Médard, "Capacity of time-varying channels with causal channel side information", IEEE Trans. Inf. Theory, vol. 53, no. 3, pp. 881-899, March 2007.

[13] T. M. Cover and S. Pombra, "Gaussian feedback capacity", IEEE Trans. Inf. Theory, vol. 35, no. 1, pp. 37-43, Jan. 1989.

[14] A. Lapidoth and S. Shamai (Shitz), "Fading channels: How perfect need "perfect side information" be?" IEEE Trans. Inf. Theory, vol. 48, no. 5, pp. 1118-1134, May 2002. 
[15] A. Lapidoth and S. M. Moser, "Capacity bounds via duality with applications to multiple-antenna systems on flat-fading channels" IEEE Trans. Inf. Theory, vol. 49, no. 10, pp. 2426-2467, Oct. 2003.

[16] A. Hjørungnes and D. Gesbert, "Complex-valued matrix differentiation: Techniques and key results," IEEE Trans. Signal Process., vol. 55, no. 6, pp. 2740-2746, Jun. 2007.

[17] N. Batir, "Inequalities for the Gamma function", Arch. Math, no. 91, pp. 554-563, 2008.

[18] R. A. Horn and C. R. Johnson, "Matrix Analysis (2nd edition)”, Cambridge University Press, 2013.

[19] R. M. Gray, "Toeplitz and circulant matrices: A review", Found. and Trends in Commun. and Inf. Theory, vol. 2, no. 3, pp. 155-239, 2006.

[20] T. M. Cover and J. A. Thomas, "Elements of information theory (2nd edition)", John Wiley and Sons, Inc., 2006.

[21] R. G. Gallager, "Information Theory and Reliable Communications", John Wiley \& Sons, Inc., New York, 1968. 